\title{
Design and Control of Unidirectional DC/DC Modular Multilevel Converter for Offshore DC Collection Point: Theoretical Analysis \& Experimental Validation
}

\author{
He Liu, Mohamed Dahidah, Senior Member, IEEE, James Yu, R. T. Naayagi, Senior Member, IEEE, and \\ Matthew Armstrong
}

\begin{abstract}
This paper presents the design and control of an advanced unidirectional DC/DC Modular Multilevel Converter (MMC) which enables the integration of off-shore windfarms with the High-Voltage Direct Current (HVDC) transmission system. The proposed converter consists of a single-phase MMC inverter, coupled with series-connected rectifier modules through a medium frequency transformer of multiple secondary windings. The modularity feature of the proposed converter enables scalability for different voltage levels. In addition to the galvanic isolation, the transformer also provides stepping gain to the output voltage. The proposed converter shows superior performance in terms of efficiency, losses and devices utilization, when compared with the most competitive unidirectional cascaded DC/DC converters such as input series output series (ISOS) and input parallel output series (IPOS). Furthermore, unlike the conventional $d-q$ control method, which involves multiple transformations, this paper employs a simple proportional resonant (PR) control strategy that directly acts on the AC output of the MMC, under the stationary reference frame. The analytical design along with the simulation and experimentally validated results, confirmed the excellent performance of the proposed converter.
\end{abstract}

Index Terms-Modular multilevel converter, DC/DC converter, DC collection point, off-shore windfarm, generalized stationary frame regulators, unidirectional DC/DC converters.

\section{INTRODUCTION}

High voltage direct current (HVDC) transmission system is a well-established and proven technology for delivering large-scale energy over a long distance with less power losses and lower reactive power requirements [1]. Large-scale offshore wind energy is increasingly growing and the interconnection between multiple farms becomes more challenging. Medium-voltage DC collection networks are a promising technology for such integration aiming to eliminate the extra conversion stages and improve the system reliability [2]. High-voltage high-power DC/DC converters are the key enabler for the DC grid. Various converter topologies have been investigated and reported in the literature, which can be broadly classified as combined (consisting of multiple converter modules) and modular multilevel topologies [3].

Most notably, Dual Active Bridge (DAB) or Single Active Bridge $(\mathrm{SAB})$ converter has received a great attention from the research community due to its distinctive features, such as: galvanic isolation, bidirectional power flow and ability to operate with high switching frequency [4]. However, the high-voltage and high-power requirements for the $\mathrm{DC} / \mathrm{DC}$ converter based HVDC systems, necessitate series and/or parallel combinations at both, the power semiconductor devices and converter modules levels [5]. Furthermore, as the requirement for offshore $\mathrm{DC}$ collection point is to deliver a high-voltage, facilitating the connection with HVDC transmission system, the input parallel output series (IPOS) configuration is commonly preferred [6], which is retained for this work as well. There are several papers investigated $\mathrm{SAB} / \mathrm{DAB}$ converter as DC collection point for the HVDC system [7-12]. However, for such a converter, the full soft switching operation can only be achieved with a limited load and input voltage range, which substantially limits the efficiency and the performance of the converter due to the increased switching losses and electromagnetic interference [9].To address this problem, an external large resonant inductor is usually connected in series with the transformer to extend the soft switching range, but the large inductance has a detrimental effect on the performance of the converter since it results in increased duty cycle losses, as well as a severe voltage ringing due to the resonance between the inductance and the junction capacitance in the converter [10]. The concept of using a saturation inductor instead of linear inductor has been discussed in [11], which effectively extends the soft switching range with lower conduction losses and without a significant duty cycle loss. However, a large core is required for thermal dissipation, limiting the whole system power density and large-scale applications. In addition, more recently improved soft switching range was achieved by additional active switches [13-15]. However, the added switches complicate the control and increase the switching losses, especially for a larger-scale system.

Modular Multilevel Converters (MMC) based DC collection point for HVDC system have received great attention in the recent years due to their manifold advantages including: modularity, straightforward scalability, and high-quality output voltage with low harmonic distortion, etc. A dual active MMC based DC/DC converter linked by a medium frequency transformer, functioning as DC collection point in the HVDC system is proposed in [16-19]. Another variation of the modular multilevel converter or else known as Alternate Arm Converter (AAC) has been also reported in [20]. These converters feature bidirectional power flow and mostly operate with medium frequency aiming to reduce the switching losses and the size/volume. However, bidirectional power transmission capability is unnecessary for the proposed offshore windfarm DC collection point, as it contributes to more switching losses and increases the control complicity.

Other variation of high-voltage $\mathrm{DC} / \mathrm{DC}$ converters have been also reported in [21-23], where in [22] an LCL based $\mathrm{DC} / \mathrm{DC}$ converter is developed using thyristor technology. While it has the capability of bidirectional power flow and DC fault clearance on both sides of the converter, however, the conversion ratio of such a converter is limited, which makes it improper for a large-scale HVDC system. Reference [23] introduces a multi-module high-gain and high-voltage transformer-less DC/DC converter using a single-switch and 
a single-inductor. However, due to the absence of the transformer, this converter lacks electrical isolation feature. On the other hand, a high-efficiency, step-up resonant DC/DC converter for offshore wind farm HVDC system is studied in [24]. The soft-switching technique is applied for all switches in the converter, therefore, the high switching frequency can be used resulting in a smaller volume and weight with lower switching losses. Nevertheless, the lack of modularity, not only limits the system from flexible scalability but also increases control complexity and manufacturing cost.

The research in this paper aimed at alleviating the abovementioned issues by proposing a unidirectional high voltage modular $\mathrm{DC} / \mathrm{DC}$ converter, employing $\mathrm{MMC}$ at the primary side of a medium frequency transformer. It is noting that the medium voltage obtained from the medium voltage DC/DC converter as shown in Fig. 1 are normally in the range of $10 \mathrm{kV}$ to $50 \mathrm{kV}$ or even higher, dependents on the technology and the layout of the offshore wind farms. In such medium voltage range, the employed MMC at primary side of the proposed converter will not require a large number of submodules, which in turns, significantly reduce the cost and losses, hence higher efficiency. Furthermore, due to modular design of MMC, the number of submodules can be flexibly changed to meet different input voltage levels requirement. Meanwhile, the DC voltage is collected at the secondary side through series-connected diode-bridge rectifier modules. Due to the use of diode bridge rectifier modules at the secondary side, the control system is not required. From the compassion in Section IV, the proposed converter shows a superior performance in terms of efficiency, losses and devices utilization, when compared with the most competitive unidirectional cascaded ISOS and IPOS converters, which makes it more attractive for this particular application. A control method based on proportional resonant (PR) strategy is employed for the proposed converter. It should be noted that the proposed converter is just intended for the high voltage DC collection point (HV DC/DC converter) as shown in Fig, 1 (red dashed line).

The rest of the paper is organized as follows: Section II describes the circuit configuration of the proposed DC/DC converter and its operating principle. The power balance analysis of the MMC at the primary side is presented in Section III. Section IV details the losses calculation of the proposed converter and its medium frequency transformer. A comparison between the proposed converter and the most competitive unidirectional topology based on Single Active Bridge (SAB) DC/DC converters with ISOS and IPOS is also presented in Section IV. A simple control strategy based on the stationary reference frame, using PR controller is derived in Section V. Section VI illustrates selected simulation and experimentally validated results. Finally, the work is concluded in Section VII.

\section{PROPOSED DC/DC CONVERTER BASED SYSTEM}

\section{A. Structure of the proposed converter}

Fig. 1 shows a simplified schematic diagram of a typical HVDC offshore windfarm using the proposed modular $\mathrm{DC} / \mathrm{DC}$ converter, functioning as high voltage (HV) DC collection point (red dashed line in Fig.1), where a singlephase (two-leg) MMC inverter producing a controllable AC voltage is connected at the primary side of a medium frequency $(400 \mathrm{~Hz})$ transformer. The DC output voltage is obtained through series-connected full-bridge rectifier modules at the multi-winding secondary side of the transformer. It is worth noting that the design is fully modular at both sides and can be easily expanded as required by simply adding more modules. The DC output voltage is controlled via controlling the AC voltage of the MMC at the primary side and a PR regulator is employed in this paper to perform the overall control of the converter.

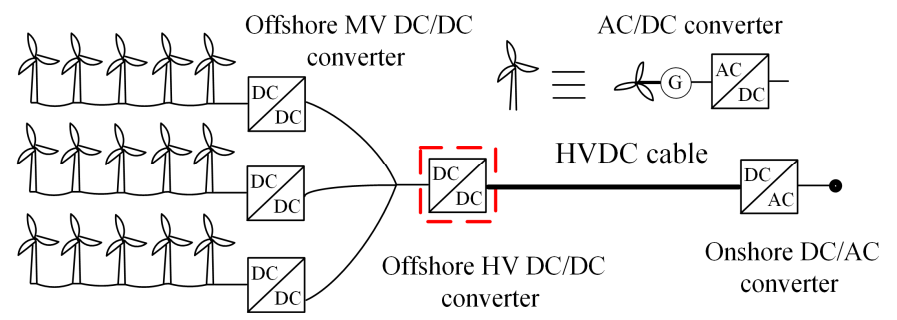

(a)

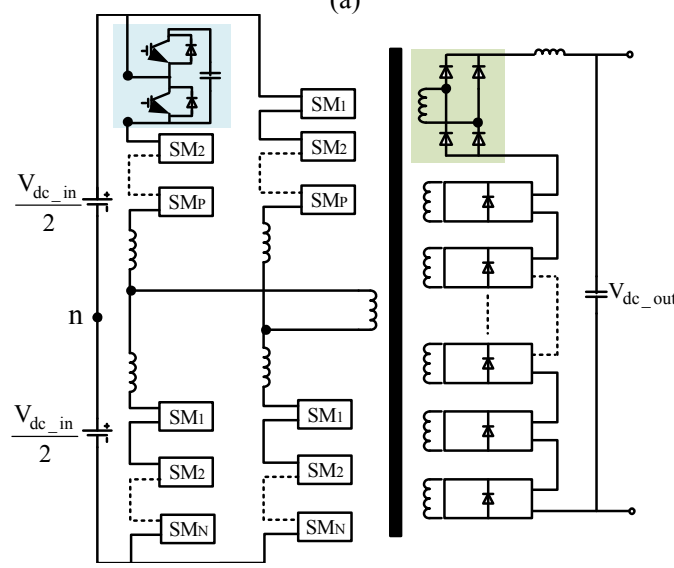

(b)

Fig.1. (a)Typical schematic diagram of off-shore HVDC transmission system and (b) using the proposed high voltage (HV) DC/DC converter functioned as DC collection point (red dashed line).

\section{B. Mathematical model of the proposed converter}

Fig. 2 shows the equivalent circuit of one-leg (Phase A) of the $\mathrm{MMC}$, where $V_{d c_{\_} \text {in }}$ and $I_{d c_{-} \text {in }}$ are the converter's DC input voltage and current, respectively. $V_{a p}$ and $V_{a N}$ are the upper and lower arm voltages of the cascede submodules of Phase A leg, respectively. $I_{a P}$ and $I_{a N}$ are the current of the upper and lower arms, respectively. $E_{a}$ is the equivalent output phase voltage as shown in Fig.2(b) and $V_{a}$ is output AC voltage, respectively. $I_{c i r}$ and $I_{a}$ are circulating current and ouput AC current, respectively.

From Fig.2, the upper and lower arm currents of Phase A leg can be expressed as:

$$
\begin{gathered}
I_{a P}=I_{a} / 2+I_{c i r} \\
I_{a N}=-I_{a} / 2+I_{c i r}
\end{gathered}
$$

where the circulating current, $I_{\text {cir }}$ is flowing through both the upper and lower amrs.

It should be noted that the circulating current has no effect on the ouptut phase current and can be expressed as:

$$
I_{\text {cir }}=\left(I_{a P}+I_{a N}\right) / 2
$$

With reference to (1) and (2), the equation of output $\mathrm{AC}$ current $I_{a}$ can be expressed in terms of upper and lower arm currents as:

$$
I_{a}=I_{a P}-I_{a N}
$$

Considering $n$ as the neutral point, applying the Kirchhoff 


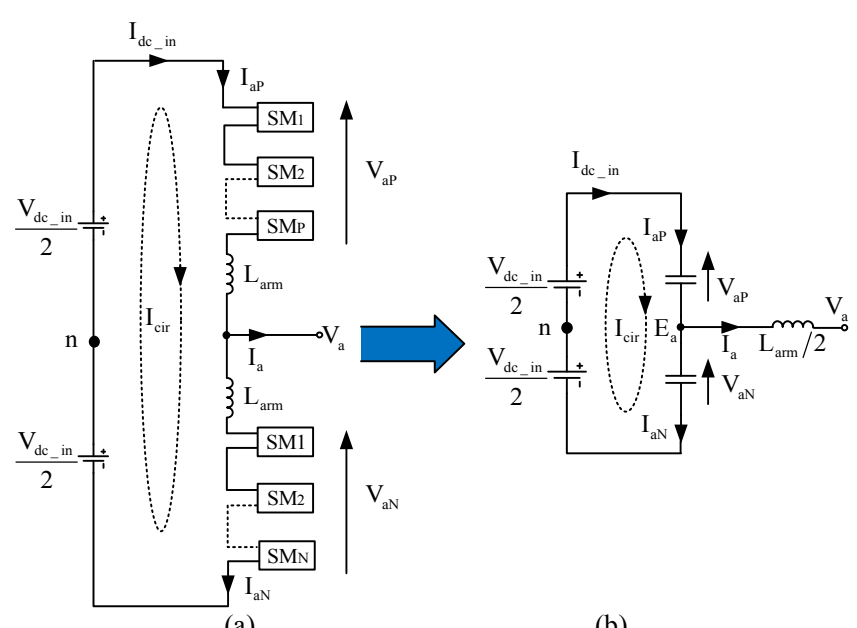

(a)

(b)
Fig.2. (a) Schematic diagram of the one-leg MMC and (b) its equivalent circuit.

Voltage Laws (KVL) for the schematic diagram of one-leg MMC as shown in Fig.2(a), therefore, the upper and lower voltages can be derived as:

$$
\begin{aligned}
& V_{a P}=\frac{V_{d c}}{2}-V_{a}-L_{a r m} \frac{d I_{a P}}{d t} \\
& V_{a N}=\frac{V_{d c}}{2}+V_{a}-L_{a r m} \frac{d I_{a N}}{d t}
\end{aligned}
$$

Combining (5) and (6), the output phase voltage $V_{a}$ can be expressed as:

$$
V_{a}=\frac{1}{2}\left(V_{a N}-V_{a P}\right)-\frac{1}{2} L_{a r m} \frac{d\left(I_{a N}-I_{a P}\right)}{d t}
$$

Substituting (4) into (7), the equivalent output phase voltage $E_{a}$ can be given by:

$$
E_{a}=\frac{1}{2}\left(V_{a N}-V_{a P}\right)=V_{a}+\frac{1}{2} L_{a r m} \frac{d I_{a}}{d t}
$$

Therefore, the mathmatical model of the one-leg MMC can be derived by rearranging (8) as:

$$
\frac{1}{2} L_{\text {arm }} \frac{d I_{a}}{d t}=E_{a}-V_{a}
$$

According to (9), the equivalent circuit of Phase A can be expressed as Fig.2 (b).

Similarly, the mathmatical model of the second leg of MMC (i.e. phase B), can be given by:

$$
\frac{1}{2} L_{\text {arm }} \frac{d I_{b}}{d t}=E_{b}-V_{b}
$$

where $E_{b}$ and $V_{b}$ are the equivalent output phase voltage and output AC voltage of Phase $\mathrm{B}$, respectively and $I_{b}$ is the ouptut AC current of Phase B.

Combining (9) and (10), the mathmatical model of single phase (two-leg) MMC can be expressed as:

$$
\frac{1}{2} L_{\text {arm }} \frac{d I_{a}}{d t}-\frac{1}{2} L_{a r m} \frac{d I_{b}}{d t}=\left(E_{a}-E_{b}\right)-\left(V_{a}-V_{b}\right)
$$

For simplicity, let:

$$
\begin{aligned}
& E_{a b}=E_{a}-E_{b} \\
& V_{a b}=V_{a}-V_{b}
\end{aligned}
$$

Hence, (11) can be re-written as:

$$
\frac{1}{2} L_{a r m} \frac{d\left(I_{a}-I_{b}\right)}{d t}=E_{a b}-V_{a b}
$$

For a single phase (two-leg) MMC, the relationship between the output current of Phase A and B can be expressed as :

$$
I_{P}=I_{a}=-I_{b}
$$

where $I_{P}$ is the transformer primary current of the proposed converter.

Substituting (15) into (14), yields:

$$
L_{a r m} \frac{d I_{P}}{d t}=E_{a b}-V_{a b}
$$

where the $E_{a b}$ and $V_{a b}$ can be considered as the equivalent primary voltage and primary terminal voltage of the transformer, respectively.

From Fig. 1 the secondary side of the transformer is made of a combination of individual and isolated rectifier modules. This can be regarded as a series connection of voltage sources $\left(V_{s 1}, V_{s 2}, \ldots, V_{s\left(N_{s}\right)}\right)$. Therefore, the total equivalent voltage at the secondary side of the transformer $V_{S}$, can be expressed as:

$$
V_{s}=V_{s 1}+V_{s 2}+\cdots+V_{s\left(N_{s}\right)}
$$

where $N_{S}$ is the number of rectifier modules at the secondary side of the transformer.

If the equivelant primary-to-secondary winding turns ratio is $R_{t}$ and the turns ratio of primary to each individual secondary winding is $T_{r}$, the equivelant secondary volatge $V_{s}$ when it is referred to the primary side can then be given by:

$$
V_{a b}=V_{s} R_{t}=V_{s 1} \frac{T_{r}}{N_{s}}+V_{s 2} \frac{T_{r}}{N_{s}}+\cdots+V_{s\left(N_{s}\right)} \frac{T_{r}}{N_{s}}
$$

Substituting (16) into (18), and with the transformer's leakage inductance $L_{k}$ referred to the primary side, the primary referred equivalent circuit of the proposed converter converter can be expressed by (19) and schematically represented by Fig.3.

$$
\left(L_{a r m}+L_{k}\right) \frac{d I_{p}}{d t}=E_{a b}-V_{s} R_{t}
$$

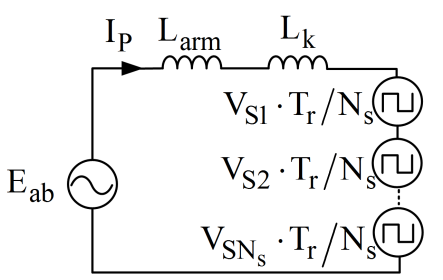

Fig.3. The equivalent circuit of the proposed converter referred to primary side.

\section{Voltage and current key-waveforms of the proposed converter}

For the sake of simplicity and easy understanding, the following are assumed for the MMC at the primary side. 1) The submodules' capacitor voltages are balanced and ripplefree. 2) The converter is operating with a unity modulation index. 3) High number of submodules, resulting in a very close to sinusoidal AC output waveform. Furthermore, the well-known carrier-phase-shift pulse width modulation (CPS-PWM) [25] technique is used in this work to modulate the MMC.

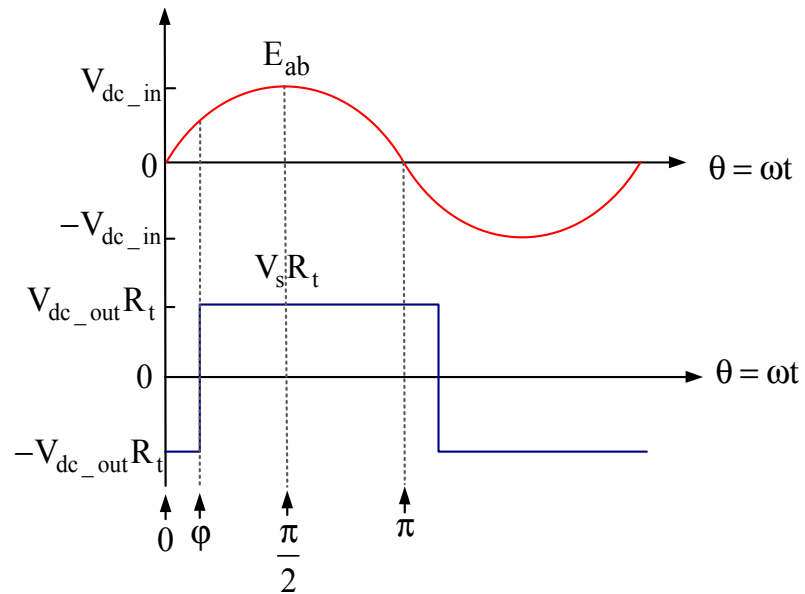

Fig.4. Typical voltage and current key waveforms of the equivalent circuit of the proposed converter with all voltages are referred to primary.

With the above assumptions, the ideal primary and secondary referred voltage waveforms of the proposed converter can be represented as in Fig.4, where the power is transferred from $V_{d c_{-} \text {in }}$ to $V_{d c_{-} o u t}$ i.e. unidirectional power 
flow. As discussed in Section II-B above, $V_{S}$ is the equivalent secondary $\mathrm{AC}$ voltage, which is equal to $V_{S}=V_{S 1}+V_{S 2}+$ $\cdots+V_{S\left(N_{S}\right)}$ and $\varphi$ is the phase shift angle by which the primary equivalent $\mathrm{AC}$ voltage, $E_{a b}$, leads the secondary equivalent $\mathrm{AC}$ voltage, $V_{S}$.

\section{Output power of the proposed converter}

It should be noted that in the analysis below takes the following two assumptions into account: 1) the AC output waveform is symmetrical and therefore, only half of the cycle is considered for the output power derivation. 2) for simplicity, the peak value of $E_{a b}$ and $V_{S}$ are equal to $V_{d c_{-} i n}$, and $V_{d c_{-} \text {out }}$, respectively, (i.e. ignoring any voltage drop across the circuit components).

From Fig.4, the output power can be derived based on the following operational intervals:

Interval $1(0 \leq \theta<\varphi)$ :

As it can be noticed from Fig.4, during this interval $V_{S}(\theta)$ is equal to the $-V_{d c_{-} o u t}$, and its primary refered voltage is $-V_{d c_{-} \text {out }} R_{t}$. Therefore, $E_{a b}(\theta)$ and $I_{P}(\theta)$ can be expressed as:

$$
\begin{gathered}
E_{a b}(\theta)=V_{d c_{-} i n} \sin (\theta) \\
I_{P}(\theta)=I_{P}(0)+\frac{1}{\omega L} \int_{0}^{\theta}\left[E_{a b}(\theta)-R_{t} V_{S}(\theta)\right] d \theta
\end{gathered}
$$

replacing $V_{S}(\theta)$ by $-V_{d c_{-} \text {out }}$ and substituting (20) into (21), yields:

$$
I_{P}(\theta)=I_{P}(0)+\frac{1}{\omega L}\left[2 V_{d c_{-} \text {in }} \sin \left(\frac{\theta}{2}\right)^{2}+R_{t} V_{d c_{-} \text {out }} \theta\right]
$$

According to (22), when $\theta=\varphi$, one can get:

$$
I_{P}(\varphi)=I_{P}(0)+\frac{1}{\omega L}\left(V_{d c_{-} \text {in }}-V_{d c_{-} \text {in }} \cos \varphi+V_{d c_{-} \text {out }} R_{t} \varphi\right)
$$

According to $V_{S}(\theta)$ and (22), the output energy during this interval can be obtained by:

$$
\begin{aligned}
\mathrm{E}_{1}= & \int_{0}^{\varphi} V_{S}(\theta) * I_{P}(\theta) d \theta=-\frac{R_{t}^{2} V_{d c_{\_} \text {out }}{ }^{2} \varphi^{2}}{2 \omega L}- \\
& V_{d c_{-} \text {out }} R_{t} \varphi I_{P}(0)-\frac{R_{t} V_{d c_{\_} \text {in }} V_{d c_{\_} \text {out }}[\varphi-\sin (\theta)]}{\omega L}
\end{aligned}
$$

Interval $2(\varphi \leq \theta<\pi)$ :

During this interval, $V_{S}(\theta)$ is equal to $V_{d c_{-} \text {out }}$ and $E_{a b}(\theta)$ remains equal to $V_{d c_{-} i n} \sin (\theta)$, therefore, $I_{P}(\theta)$ can be expressed as:

$$
I_{P}(\theta)=I_{P}(\varphi)+\frac{1}{\omega L} \int_{\varphi}^{\theta}\left[E_{a b}(\theta)-R_{t} V_{S}(\theta)\right] d \theta
$$

Substituting $V_{S}(\theta)=V_{d c_{-} \text {out }}, E_{a b}(\theta)=V_{d c_{-} \text {in }} \sin (\theta)$ and (23) into $I_{P}(\theta)$ with (25) yields,

$$
\begin{gathered}
I_{P}(\theta)=I_{P}(0)+\frac{1}{\omega L}\left[V_{d c_{-} \text {in }}-V_{d c_{-} \text {in }} \cos \varphi-\right. \\
\left.R_{t} V_{d c_{-} \text {out }} \theta+2 R_{t} V_{d c_{-} \text {out }} \varphi\right]
\end{gathered}
$$

From (26), at $\theta=\pi$, one can get:

$$
I_{P}(\pi)=I_{P}(0)+\frac{1}{\omega L}\left[2 V_{d c_{-} \text {in }}-\pi R_{t} V_{d c_{-} \text {out }}+2 R_{t} V_{d c_{-} \text {out }}\right]
$$

Similarly, the transferred energy during this interval is given by:

$$
\begin{gathered}
\mathrm{E}_{2}=\int_{\varphi}^{\pi} V_{S}(\theta) * I_{P}(\theta) d \theta=-\frac{R_{t} V_{d c_{-} \text {out }}}{2 \omega L} \\
{\left[\left(\begin{array}{c}
2 V_{d c_{-} \text {in }} \varphi-2 \pi V_{d c_{-} \text {in }}-2 V_{d c_{-} \text {in }} \sin (\theta)+\pi^{2} R_{t} V_{d c_{-} \text {out }} \\
+3 R_{t} V_{d c_{-} \text {out }} \varphi^{2}-2 \pi L I_{P}(0)+2 L \varphi I_{P}(0)-4 \pi R_{t} V_{d c_{-} \text {out }} \varphi
\end{array}\right)\right]}
\end{gathered}
$$

Therefore, from (24) and (28), the ouput power of the proposed converter can be calcultaed as:

$$
P_{\text {out }}=\frac{E_{1}+E_{2}}{\pi}
$$

Owing to the half-cycle symmetry, $I_{P}(0)=-I_{P}(\pi)$. Therefore, according to (27), the initial current $I_{P}(0)$ can be calculated as:

$$
I_{P}(0)=\frac{2 V_{d c_{\_} \text {in }}-\pi R_{t} V_{d c_{-} \text {out }}+2 R_{t} V_{d c_{\_} \text {out }} \varphi}{2 \omega L}
$$

Therefore, the ouput power of the proposed converter at any phase shift angle, $\varphi$, can be expressed by:

$$
P_{\text {out }}(\varphi)=\frac{2 G V_{d c_{-} i n}^{2} \sin (\varphi)}{\pi \omega L}
$$

where $G$ is defined as the primary-referred DC voltage gain of the proposed converter which is equal to $\mathrm{G}=\frac{V_{d c_{\text {oout }} R_{t}}}{V_{d c_{-} \text {in }}}$, which is often known as the DC conversion ratio [26].

\section{E. Output power characteristics of the proposed converter}

Similar to the conventional Single Active Bridge (SAB) DC/DC converter [27], pahse shift angle $\varphi$ is the point where the primary current, $I_{P}$ crosses the zero, which means $I_{P}(\varphi)=0$. Using this relationship $\left(I_{P}(\varphi)=0\right)$ gets:

$$
G=\frac{2 \cos (\varphi)}{\pi}
$$

Substituting (32) into (31), yields:

$$
P_{\text {out }}(\varphi)=\frac{4 V_{d c \_ \text {in }}^{2} \sin (\varphi) \cos (\varphi)}{\pi^{2} \omega L}
$$

It should be noted that (33) is derived based on the assumption of the modulation index $M=1$, which corresponds to the maximum power transfer capability of the proposed converter.

For simplicity, the ouput power is normalized to a base power of $P_{b}=\frac{4 V_{d c_{-} i n}^{2}}{\pi^{2} \omega L}$, which results in:

$$
P_{\text {out_norm }}(\varphi)=M^{2} \sin (\varphi) \cos (\varphi)
$$

where modulation index $M \in[0,1]$ is introduced in oder to get the generalized equation of the ouput power.

Fig. 5 illustrates the variation of the normalized power of (34) with respect to phase shift angle $\varphi$ (noting that the solid line represents theoretical results and dashed line represents simulation results based on parameters in Table V. Furthermore, it is also clear from the same figure that the power transfer capability of the proposed converter is influenced by the modulation index $M$, and the phase shift angle $\varphi$, where the highest power is achieved with a unity modulation index. In theory, the maximum output power occurs at the point of $\frac{d P_{\text {out_norm }}(\varphi)}{d \varphi}=0$, for which $\varphi=\frac{\pi}{4}$. However, when taking losses of circuit and primary AC voltage (non ideal sinusoidal) into considersion, the maximum output power point will deviate from $\varphi=\frac{\pi}{4}$ as shown in Fig.5.

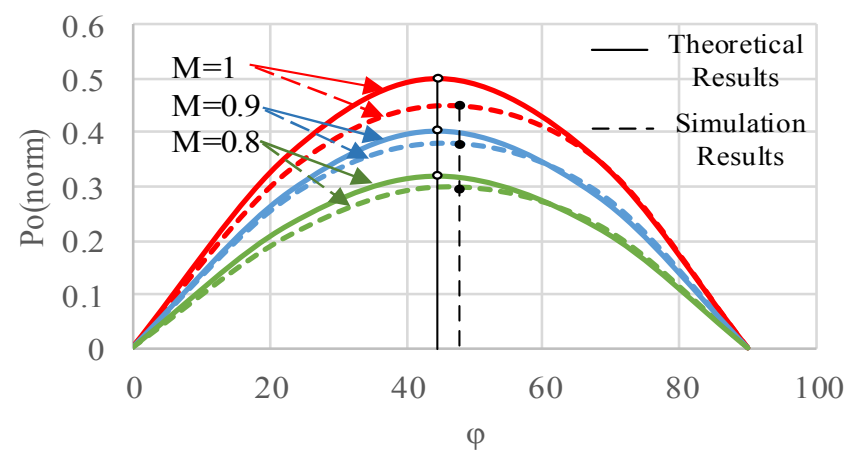

Fig.5. Normalized output power versus pahse shift angle $\varphi$ of the proposed converter. 


\section{Power Balance oF The Proposed CONVERTER}

One of the most challenging aspect of MMC is how to keep the submodule capacitors voltage controlled. Therefore, it is very important to analyze and understand the stored energy and the power flow between the submodules and/or the converter arms [28-29]. To simplify the analysis, the following are assumed: 1) the converter's arm inductance are neglected. 2) only the fundamental component of the MMC $\mathrm{AC}$ voltage is considered. 3) only one-leg of MMC converter is considered as example. Therefore, the output $\mathrm{AC}$ current and voltage of Phase A can be approximately expressed as:

$$
\begin{gathered}
V_{a}=\widehat{V}_{m} \sin (\omega t) \\
I_{a}=\hat{I}_{m} \sin (\omega t-\emptyset)
\end{gathered}
$$

where $V_{a}$ and $\widehat{V}_{m}$ are the instantaneous output AC voltage and its peak value, respectively; $I_{a}$ and $\hat{I}_{m}$ are the instantaneous output AC current and its peak value, respectively.

The equivalent circuit of a balanced one-leg MMC is shown in Fig.6 (a), where the upper and lower submodules are modeled as a voltage source. Furthermore, an unbalanced energy across the upper and lower arm causing a DC offset to the output AC voltage, is presented by a DC source denoted by $k V_{d c_{-} i n}$ (i.e. $k$ is defined as unbalance factor, $\mathrm{k} \in$ $[-0.1,0.1])$ in the upper and lower arms as shown in Fig.6 (b).

Therefore, applying KVL, the upper and lower arms voltages can respectively be defined by (37) and (38).

$$
\begin{aligned}
& V_{a P}=\frac{V_{d c_{\_} i n}}{2}-\widehat{V}_{m} \sin \omega t+k V_{d c_{\_} i n} \\
& V_{a N}=\frac{V_{d c_{\_} i n}}{2}+\widehat{V}_{m} \sin \omega t-k V_{d c_{\_} i n}
\end{aligned}
$$

Let $M$ be the modulation index of the MMC output AC voltage. Therefore, the peak output voltage of one-leg can be then given by:

$$
\widehat{V}_{m}=\frac{1}{2} M V_{d c_{-} i n}
$$

Rearranging (39), the input DC voltage can be expressed as:

$$
V_{d c_{-} i n}=\frac{2 \widehat{V}_{m}}{M}
$$

Substituting (40) into (37) and (38) yields:

$$
\begin{aligned}
& V_{a P}=\frac{\widehat{V}_{m}}{M}-\widehat{V}_{m} \sin \omega t+k \frac{2 \widehat{V}_{m}}{M} \\
& V_{a N}=\frac{\widehat{V}_{m}}{M}+\widehat{V}_{m} \sin \omega t-k \frac{2 \widehat{V}_{m}}{M}
\end{aligned}
$$

It is well-known that DC voltage offset will produce a DC current, denoted by $I_{k i}$ in the upper and lower arms, which is caused by the effect of the unbalanced energy. From Fig.6 (b), the upper and lower arms currents can be defined as:

$$
\begin{aligned}
& I_{a P}=I_{d c_{-} i n}+\frac{1}{2} \hat{I}_{m} \sin (\omega t-\emptyset)-I_{k i} \\
& I_{a N}=I_{d c_{-} i n}-\frac{1}{2} \hat{I}_{m} \sin (\omega t-\emptyset)+I_{k i}
\end{aligned}
$$

As the introduced unbalanced voltage sources are only DC, consequently, the resultant currents are DC as well. Furthermore, this current flow through the load impedance, which is naturally inductive, however only the resistive part of the impedance needs to be considered as the current is a DC. Therefore, the unbalance DC current $I_{k i}$ in the circuit can therefore be calculated by:

$$
I_{k i}=\frac{k V_{d c_{-} i n}}{R}
$$

where $R$ is the load resistance.

Neglecting the power losses in the circuit, the power flowing from $\mathrm{DC}$ side is equal to the power consumed at the $\mathrm{AC}$ side of MMC. Hence,

$P_{d c}=P_{a c}=V_{d c_{\_} i n} I_{d c_{-} i n}=\frac{1}{2} \hat{V}_{m} \hat{I}_{m} \cos \emptyset+2 I_{k i} \cdot k V_{d c_{-} i n}(46)$ where $V_{d c}$ and $I_{d c}$ are the input DC voltage and current, respectively; $\cos \emptyset$ is the power factor. It is important to note

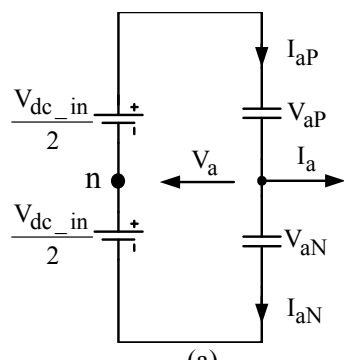

(a)

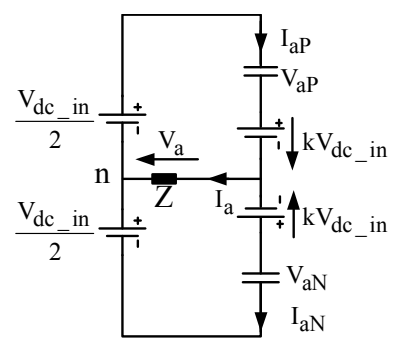

(b)
Fig.6. Equivalent circuit of one-leg MMC (a) balanced energy condition (b) Unbalanced energy condition

that the consumed power, $2 I_{k i} \cdot k V_{d c}$ is caused by biased DC on the AC load.

By substituting $\widehat{V}_{m}$ of (39) and $I_{k i}$ of (45) into (46), the input DC current can be obtained by:

$$
I_{d c_{-} \text {in }}=\frac{1}{4} M \hat{I}_{m} \cos \emptyset+\frac{4 k^{2} \widehat{V}_{m}}{R \cdot M}
$$

Now, substituting (39), (45) and (47) into (43) and (44) yields:

$$
\begin{aligned}
& I_{a P}=\frac{1}{4} M \hat{I}_{m} \cos \emptyset+\frac{4 k^{2} \widehat{V}_{m}}{R \cdot M}+\frac{1}{2} \hat{I}_{m} \sin (\omega t-\emptyset)-\frac{2 k \widehat{V}_{m}}{M} \\
& I_{a N}=\frac{1}{4} M \hat{I}_{m} \cos \emptyset+\frac{4 k^{2} \widehat{V}_{m}}{R \cdot M}-\frac{1}{2} \hat{I}_{m} \sin (\omega t-\emptyset)+\frac{2 k \widehat{V}_{m}}{M}
\end{aligned}
$$

From the above analysis, the instantaneous power of the upper and lower arms is expressed by:

$$
\begin{aligned}
P_{a P}= & V_{a P} \times I_{a P}=\left(\frac{\widehat{V}_{m}}{M}-\widehat{V}_{m} \sin \omega t+k \frac{2 \widehat{V}_{m}}{M}\right) . \\
& {\left[\frac{1}{4} M \hat{I}_{m} \cos \emptyset+\frac{4 k^{2} \widehat{V}_{m}}{R \cdot M}+\frac{1}{2} \hat{I}_{m} \sin (\omega t-\emptyset)-\frac{2 k \widehat{V}_{m}}{M}\right](50) } \\
P_{a N}= & V_{a N} \times I_{a N}=\left(\frac{\widehat{V}_{m}}{M}+\widehat{V}_{m} \sin \omega t-k \frac{2 \widehat{V}_{m}}{M}\right) . \\
& {\left[\frac{1}{4} M \hat{I}_{m} \cos \emptyset+\frac{4 k^{2} \widehat{V}_{m}}{R \cdot M}-\frac{1}{2} \hat{I}_{m} \sin (\omega t-\emptyset)+\frac{2 k \widehat{V}_{m}}{M}\right](51) }
\end{aligned}
$$

By integrating (50) and (51) over one fundamental period, one can obtain the energy variation in the upper and lower arms as follows:

$$
\begin{gathered}
E_{a P}=\int_{0}^{2 \pi} P_{a P} d t=\frac{\pi \widehat{V}_{m}}{R}\left(\frac{16 \widehat{V}_{m} k^{3}}{M^{2}}+k \hat{I}_{m} R \cos \emptyset-\frac{4 \widehat{V}_{m} k}{M^{2}}\right)(52) \\
E_{a N}=\int_{0}^{2 \pi} P_{a N} d t=-\frac{\pi \widehat{V}_{m}}{R}\left(\frac{16 \widehat{V}_{m} k^{3}}{M^{2}}+k \hat{I}_{m} R \cos \emptyset-\frac{4 \widehat{V}_{m} k}{M^{2}}\right)
\end{gathered}
$$

Equations (52) and (53) describe the effect of the unbalance factor $k$ on the arm energy, where in the case of $k=0$, the average energy transferred to the upper and lower arms over one cycle is zero, i.e. $E_{a P}=E_{a N}=0$. However, when $\mathrm{k} \neq 0$, although the transferred energy in one cycle between upper and lower arms remains zero, i.e. $E_{a P}+E_{a N}=0$; but there will be an unbalanced energy, i.e. $E_{a P}=E_{a N} \neq 0$, which causes deviations in submodules capacitor voltage and therefore energy exchange between the upper and lower arms is necessary to balance. Furthermore, as the circulating current influences the charging and discharging of submodule capacitors, therefore it is mandatory to control the circulating current to achieve voltage balance. A voltage balance control strategy which includes submodule voltage control and arm average voltage control, is employed for the proposed converter. Since this control method has been welldocumented [30], therefore, no further details are given here.

\section{LOSSES CALCULATION AND TOPOLOGICAL COMPARISON}

It is very important to demonstrate the superiority of the proposed converter compared to its competitive unidirectional DC/DC converters. For such applications high output voltage is required, therefore, cascaded topologies such as input series output series (ISOS) and 


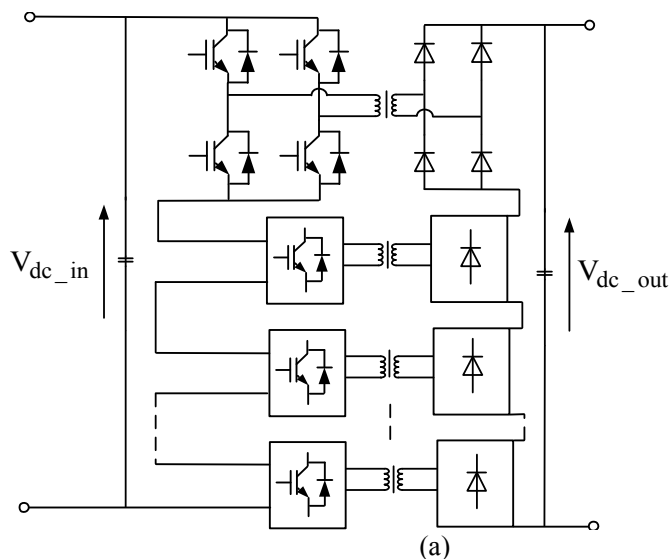

(a)



(b)

Fig.7. Unidirectional cascaded DC/DC converters (a) input series output series (ISOS) (b) input parallel output series (IPOS).

input parallel output series (IPOS) as shown in Fig.7 are selected for comparison with the proposed converter.

To simplify the analysis, it should be noted that 1 ) only the fundamental component of the AC output current of the converter is considered for the power loss calculation. 2) The power is equally shared between the SAB based submodules in the unidirectional cascaded converters. Furthermore, due to the current flown characteristics of unidirectional cascaded DC/DC converters (e.g. ISOS/IPOS) has been well introduced and analyzed in [7-8], hence just taking proposed converter as example to derive its losses calculation method.

\section{A. Losses calculation of the proposed converter}

Since the proposed converter is a unidirectional, therefore, the losses are calculated only when the MMC operates as an inverter, however, it should be noted that in both modes (i.e. rectifier/inverter), the calculation process is the same. Furthermore, only the fundamental component of the $\mathrm{AC}$ output voltage of the MMC is considered in the power loss calculation.

Since CPS-PWM is utilized to control the switches of the proposed MMC converter, therefore, the duty cycle $\tau(t)$ of all switches is defined by:

$$
\tau(t)=\frac{1+M \sin (\omega t)}{2}
$$

where $M$ is the modulation index, $\omega$ is the fundamental angular frequency of the AC output waveform.

Table I lists the possible operating modes of the MMC's submodule (SM) where the currents directions are schematically presented in Fig.8 [28]. Taking the upper arm
Table I Submodule states of MMC

\begin{tabular}{|l|c|c|c|c|l|}
\hline states & $\boldsymbol{T}_{\mathbf{1}}$ & $\boldsymbol{T}_{\mathbf{2}}$ & $\boldsymbol{I}_{\boldsymbol{s m}}$ & $\boldsymbol{V}_{\boldsymbol{s m}}$ & submodule \\
\hline 1 & ON & OFF & $>0$ & $V_{c}$ & $\begin{array}{l}\text { Inserted } \\
\text { (charging) }\end{array}$ \\
\hline 2 & ON & OFF & $<0$ & $V_{c}$ & $\begin{array}{l}\text { Inserted } \\
\text { (discharging) }\end{array}$ \\
\hline 3 & OFF & ON & $>0$ & 0 & By-passed \\
\hline 4 & OFF & ON & $<0$ & 0 & By-passed \\
\hline
\end{tabular}

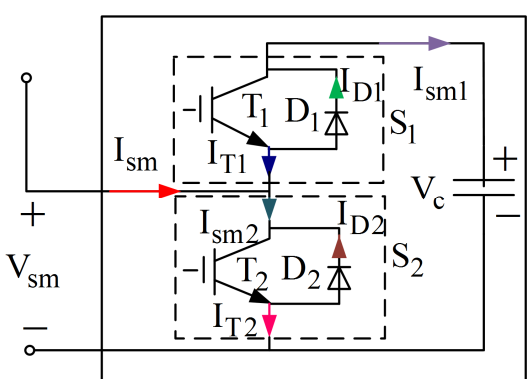

Fig. 8 Current definition of a submodule of MMC.

of Phase A as an example, each SM is either inserted or bypassed, making the voltage of Phase $\mathrm{A}$ is either increased or decreased by a SM capacitor voltage, $V_{c}$, respectively. When the modulation signal is greater than the carrier signal, $\mathrm{T}_{2}$ is turned on and $\mathrm{T}_{1}$ is turned off, which means $\mathrm{SM}$ is by-passed. However, on the other hand, when the modulation signal is less than the carrier signal, $\mathrm{T}_{2}$ is turned off and $\mathrm{T}_{1}$ is turned on, which means SM is inserted in the circuit. Therefore, if the carrier cycle is $T_{c}$, then the conduction time of $\mathrm{T}_{2}$ when the $\mathrm{SM}$ is by-passed is equal to $\tau(t) \cdot T_{c}$ according to the derived duty cycle in (54). On the other hand, the conduction time of $\mathrm{T}_{1}$ equals to $[1-\tau(t)] \cdot T_{c}$, when the $\mathrm{SM}$ is inserted. Therefore, based on above analysis, the current flowing through the upper and lower IGBT modules, $S_{1}$ and $S_{2}$, respectively can be expressed as:

$$
\begin{aligned}
& I_{s m 1}=[1-\tau(t)] * I_{a P}(t) \\
& I_{s m 2}=\tau(t) * I_{a P}(t)
\end{aligned}
$$

where $I_{a P}(t)$ is the upper arm current of Phase A.

From the operation principle of the MMC, in the normal circumstances, the input DC current, $I_{d c_{-} \text {in }}$ would be evenly distributed between the two legs, and the AC output currents of each phase are evenly distributed between the upper and the lower arms. Therefore, the upper arm current of Phase A can be expressed as:

$$
I_{a P}(t)=\frac{I_{d c_{-} i n}}{2}+\frac{1}{2} \hat{I}_{a} \sin (\omega t-\delta)
$$

Substituting (54) and (57) into (55) and (56) yields:

$$
\begin{aligned}
& I_{s m 1}=\frac{1-M \sin (\omega t)}{2} *\left[\frac{I_{d c_{\_} i n}}{2}+\frac{1}{2} \hat{I}_{a} \sin (\omega t-\delta)\right] \\
& I_{s m 2}=\frac{1+M \sin (\omega t)}{2} *\left[\frac{I_{d c_{2} i n}}{2}+\frac{1}{2} \hat{I}_{a} \sin (\omega t-\delta)\right]
\end{aligned}
$$

From (58) and (59), the effective and average values of the currents flowing through the switches and diodes of SM can be derived as follows:

1) The average current flowing through diode $D_{1}$ is:

$$
I_{D 1 \_a v g}=\frac{1}{2 \pi} \int_{\delta}^{\delta+\pi} I_{s m 1} d \omega t
$$

Substituting (58) into (60) yields:

$$
I_{D 1 \_a v g}=\frac{1}{2 \pi}\left[\frac{\hat{I}_{a}}{2}+\frac{\pi I_{d c_{\_} i n}}{4}-\frac{I_{d c_{\_} \_i n} M \cos (\delta)}{2}-\frac{\pi \hat{I}_{a} M \cos (\delta)}{8}\right]
$$

2) The square of effective current flowing through diode $D_{1}$ is :

$$
I_{D 1 \_r m s}{ }^{2}=\frac{1}{2 \pi} \int_{\delta}^{\delta+\pi} I_{s m 1}{ }^{2} d \omega t
$$


Substituting (58) into (62) yields:

$$
\begin{array}{r}
I_{D 1 \_r m s}{ }^{2}=\frac{1}{2 \pi}\left[\frac{\hat{I}_{a} I_{d c_{\_} i n}}{4}+\frac{\pi \hat{I}_{a}{ }^{2}}{32}+\frac{\pi I_{d c_{\_} i n}{ }^{2}}{36}+\frac{\hat{I}_{a} I_{d c_{\_} i n} M^{2}}{8}-\right. \\
\frac{a^{2} M \cos (\delta)}{6}-\frac{I_{d c_{i n} i n}{ }^{2} M \cos (\delta)}{4}+\frac{\pi \hat{I}_{a}{ }^{2} M^{2}}{64}+\frac{\pi I_{d c_{\_} i n}{ }^{2} M^{2}}{32}+ \\
\left.\frac{\hat{I}_{a} I_{d c_{\_} i n} M^{2} \cos (2 \delta)}{24}+\frac{\pi \hat{I}_{a}{ }^{2} M^{2} \cos (2 \delta)}{128}+\frac{\pi \hat{I}_{a} I_{d c_{i} i n} M \cos (\delta)}{8}\right]
\end{array}
$$

3) The average current flowing through switch $T_{1}$ is:

$$
I_{T 1 \_a v g}=\frac{1}{2 \pi} \int_{\delta+\pi}^{\delta+2 \pi} I_{s m 1} d \omega t
$$

Substituting (58) into (64) yields:

$I_{T 1_{\_} a v g}=-\frac{1}{2 \pi}\left[\frac{\hat{I}_{a}}{2}-\frac{\pi I_{d c_{-} i n}}{4}-\frac{I_{d c_{\_} i n} M \cos (\delta)}{2}+\frac{\pi \hat{I}_{a} M \cos (\delta)}{8}\right]$

4) The square of effective current flowing through switch

$T_{1}$ is :

$$
I_{T 1 \_r m s}{ }^{2}=\frac{1}{2 \pi} \int_{\delta+\pi}^{\delta+2 \pi} I_{s m 1}^{2} d \omega t
$$

Substituting (58) into (66) yields:

$$
\begin{array}{r}
I_{T 1_{-} r m s}{ }^{2}=\frac{1}{2 \pi}\left[\frac{\pi \hat{I}_{a}{ }^{2}}{32}-\frac{\hat{I}_{a} I_{d c_{\_} i n}}{4}+\frac{\pi I_{d c_{\_} i n}{ }^{2}}{16}-\frac{\hat{I}_{a} I_{d c_{\_} i n} M^{2}}{8}+\right. \\
\frac{\hat{I}_{a}{ }^{2} M \cos (\delta)}{6}+\frac{I_{d c_{i n} i n}{ }^{2} M \cos (\delta)}{4}+\frac{\pi \hat{I}_{a}{ }^{2} M^{2}}{64}+\frac{\pi I_{d c_{\_} i n}{ }^{2} M^{2}}{32}- \\
\left.\frac{\hat{I}_{a} I_{d c_{-} i n} M^{2} \cos (2 \delta)}{24}+\frac{\pi \hat{I}_{a}{ }^{2} M^{2} \cos (2 \delta)}{128}-\frac{\pi \hat{I}_{a} I_{d c_{\_} i n} M \cos (\delta)}{8}\right]
\end{array}
$$

5) The average current flowing through switch $T_{2}$ is :

$$
I_{T 2 \_a v g}=\frac{1}{2 \pi} \int_{\delta}^{\delta+\pi} I_{s m 2} d \omega t
$$

Substituting (59) into (68) yields:

$$
I_{T 2 \_a v g}=\frac{1}{2 \pi}\left[\frac{\hat{I}_{a}}{2}+\frac{\pi I_{d c_{\_} i n}}{4}+\frac{I_{d c_{-} i n} M \cos (\delta)}{2}+\frac{\pi \hat{I}_{a} M \cos (\delta)}{8}\right]
$$

6) The square of effective current flowing through switch $T_{2}$ is :

$$
I_{T 2 \_r m s}{ }^{2}=\frac{1}{2 \pi} \int_{\delta}^{\delta+\pi} I_{s m 2}^{2} d \omega t
$$

Substituting (59) into (70) yields:

$$
\begin{aligned}
& I_{T 2 \_r m s}{ }^{2}=\frac{1}{2 \pi}\left[\frac{\hat{I}_{a} I d c_{-} i n}{4}+\frac{\pi \hat{I}_{a}{ }^{2}}{32}+\frac{\pi I_{d c_{-} i n}{ }^{2}}{16}+\frac{\hat{I}_{a} I_{d c_{-} i n} M^{2}}{18}+\right. \\
& \frac{\hat{I}_{a}{ }^{2} M \cos (\delta)}{6}+\frac{I_{d c_{i n}{ }^{2} M \cos (\delta)}}{4}+\frac{\pi \hat{I}_{a}{ }^{2} M^{2}}{64}+\frac{\pi I_{d c_{\_} i n}{ }^{2} M^{2}}{32}+ \\
& \left.\frac{\hat{I}_{a} I_{d c_{-} i n} M^{2} \cos (2 \delta)}{224}+\frac{\pi \hat{I}_{a}{ }^{2} M^{2} \cos (2 \delta)}{128}+\frac{\pi \hat{I}_{a} I_{d c_{-} i n} M \cos (\delta)}{8}\right]
\end{aligned}
$$

7) The average current flowing through diode $D_{2}$ is :

$$
I_{D 2 \_a v g}=\frac{1}{2 \pi} \int_{\delta+\pi}^{\delta+2 \pi} I_{s m 2} d \omega t
$$

Substituting (59) into (72) yields:

$$
I_{D 2 \_a v g}=-\frac{1}{2 \pi}\left[\frac{\hat{I}_{a}}{2}-\frac{\pi I_{d c_{\_} i n}}{4}+\frac{I_{d c_{\_} i n} M \cos (\delta)}{2}-\frac{\pi \hat{I}_{a} M \cos (\delta)}{8}\right]
$$

8) The square of effective current flowing through diode $D_{2}$ is :

$$
I_{D 2 \_r m s}{ }^{2}=\frac{1}{2 \pi} \int_{\delta+\pi}^{\delta+2 \pi} I_{s m 2}^{2} d \omega t
$$

Substituting (59) into (74) yields:

$$
\begin{aligned}
& I_{D 2 \_r m s}{ }^{2}=\frac{1}{2 \pi}\left[\frac{\pi \hat{I}_{a}{ }^{2}}{32}-\frac{\hat{I}_{a} I_{d c_{-} i n}}{4}+\frac{\pi I_{d c_{-} i n}{ }^{2}}{16}-\frac{\hat{I}_{a} I_{d c_{-} i n} M^{2}}{8}-\right. \\
& \frac{\hat{I}_{a}{ }^{2} M \cos (\delta)}{6}-\frac{I_{d c_{\_} i n}{ }^{2} M \cos (\delta)}{4}+\frac{\pi \hat{I}_{a}{ }^{2} M^{2}}{64}+\frac{\pi I_{d c_{-} i n}{ }^{2} M^{2}}{32}- \\
& \left.\frac{\hat{I}_{a} I_{d c_{\_} i n} M^{2} \cos (2 \delta)}{24}+\frac{\pi \hat{I}_{a}{ }^{2} M^{2} \cos (2 \delta)}{128}+\frac{\pi \hat{I}_{a} I_{d c_{\_} i n} M \cos (\delta)}{8}\right]
\end{aligned}
$$

The conduction losses are calculated over one fundamental AC period using the above-derived average/effective currents, using:

$$
\begin{aligned}
& P_{D_{-} \text {con }}=I_{D_{\_} a v g} V_{D_{-} 0}+r_{D_{-} 0} I_{D_{-} r m s}^{2} \\
& P_{T_{-} \text {con }}=I_{T_{-} \text {avg }} V_{T_{-} 0}+r_{T_{-} 0} I_{T_{-} r m s}^{2}
\end{aligned}
$$

where $P_{D_{-} \text {con }}$ and $P_{T_{-} \text {con }}$ are the SM's diode and switch conduction losses, respectively, within one fundamental $\mathrm{AC}$ period, $V_{D_{-} 0}$ and $V_{T_{-} 0}$ are the threshold voltages of the diode and the switch, respectively, and $r_{D_{0} 0}$ and $r_{T_{0} 0}$ are the forward conduction resistance of the diode and the switch, respectively. It is worth noting that the blocking state losses are much small compared with the conduction losses, hence are ignored in this analysis.

According to [31], the turn-on/off switching losses are approximately proportional with the average current flowing through the switch; therefore, the switching losses over one fundamental $\mathrm{AC}$ period can be calculated by:

$$
\begin{aligned}
& P_{s w}=f\left(E_{\text {on }}+E_{\text {off } f}\right) \frac{V_{c} I_{T_{\_}} a v g}{V_{T_{\_} r e f} I_{T_{-} a v g}} \\
& P_{\text {rec }}=f E_{\text {rec }} \frac{V_{c} I_{D_{\_} a v g}}{V_{D_{-} r e f I_{D_{-}} a v g}}
\end{aligned}
$$

where $f$ is the switching frequency; $E_{\text {on }}$ and $E_{\text {off }}$ are the switch's turn-on and turn-off energy losses, respectively, $V_{T_{-} r e f}$ and $I_{T_{-} r e f}$ are the switch's reference voltage and current, respectively, $E_{r e c}$ is the reverse recovery energy losses of diode, and $V_{D_{-}}$ref and $I_{D_{-} r e f}$ are the diode's reference voltage and current, respectively. The values of $E_{\text {on }}, E_{\text {off }}, V_{T_{-} r e f}, I_{T_{-} a v g}, E_{\text {rec }}, V_{D_{-} r e f}$, and $I_{D_{-} r e f}$ can be obtained from the datasheet of the particular device. Similarly, the losses of diode bridge rectifier modules at the secondary side can also be calculated using (76) and (79).

\section{B. Losses evaluation of the medium frequency transformer}

In general, there are two types of losses in a transformer, copper losses and core losses. Copper losses are essentially caused by the conductor resistivity; and with the skin and proximity effects, these losses are increased with the frequency. However, using Litz wires greatly reduces these effects and therefore for this approximate analysis, the skin and proximity effects will not be considered. Hence, the copper losses, $P_{\text {copper }}$ is defined as:

$$
P_{\text {copper }}=R I_{r m s}^{2}
$$

where, $R$ is the equivalent resistance of the transformer windings and $I_{r m s}$ is the effective current flowing through these windings.

The core losses are proportionally influenced by the maximum flux density, which is an important factor in designing a transformer. However, for a given magnetic flux, the flux density is solely determined by the core crosssectional area. In general, the Steinmetz Equation [32] is used to predict the core losses, which can be expressed as follows:

$$
P_{\text {core }}=k V_{\text {core }} f^{\alpha} B_{p k}^{\beta}
$$

where $P_{\text {core }}$ and $V_{\text {core }}$ are the core losses and core volume, respectively; $B_{p k}$ is the peak core flux density and the coefficients $K, \alpha$, and $\beta$ are given by the properties of the core material. $f$ is the AC frequency of the system.

\section{Comparison between the proposed converter and unidirectional cascaded ISOS and IPOS converters}

The proposed converter aims to reduce the complexity and the losses of the DC collecting point converters of the offshore wind farms. Therefore, it is very important to evaluate its performance against other available unidirectional DC/DC converter topologies. As the natural requirement of such a converter is boost the voltage at the output side, hence, the 
proposed converter is compared with the unidirectional multimodel, SAB DC/DC converters with ISOS and IPOS configurations, reported in [5] [7-8].

Table II presents the parameters of the proposed converter and the two SAB converter systems. For a sensible comparison, both converters are rated at the same power as the proposed converter. Moreover, the three topologies utilize the same diode-bridge rectifier modules (i.e. unidirectional), connected in series at the secondary side. In this paper, fourteen diode-bridge rectifier modules are cascaded in series to produce an output DC voltage of $140 \mathrm{kV}$. It should be noted that each valve of the diode-bridge rectifier module consists of two diodes in series [33], with a voltage blocking capability of $5 \mathrm{kV}$, each. Furthermore, the unidirectional ISOS and IPOS DC/DC converters are designed with the same requirement as of the proposed converter (i.e. blocking voltage capability of each SAB based submodule at primary side is $1.07 \mathrm{kV}$ ). This necessitates a total of 56 IGBT modules for ISOS converter and 784 IGBT modules for IPOS converter at primary side as opposed to 112 IGBT modules for the proposed converter. However, it should be noted that for the conventional ISOS and IPOS converters, the number of converter modules on the primary and the secondary sides are dependent on each other. If higher voltage is required at the output side, then the same number of converter modules must be added at both side with their associated transformers, hence losses and complexity. However, with the proposed converter, these are independent of each other, where more rectifier modules can be added at the secondary side without increasing the number of IGBT modules at the primary side.

For a fair comparison, all three topologies are modulated utilizing sinusoidal pulse width modulation (SPWM) with the same carrier-reference frequency ratio, denoted by $R_{c}$ (i.e. $R_{c}=f_{C} / f_{M}$, where $f_{C}$ and $f_{M}$ are frequency of the triangular carrier and reference waveform, respectively). $400 \mathrm{~Hz}$ is selected as the $\mathrm{AC}$ fundamental frequency of all these three DC/DC converters.

The losses of topologies are also evaluated based on the analysis presented in above of Section IV to estimate the efficiency of the topologies across the whole range of the output power. For this purpose, the suitable IGBTs and Diodes for topologies must be selected. As above mentioned, for a sensible comparison, all three unidirectional converters are rated at the same power and input/output DC voltage as shown in table II. However, due to the different configurations at primary side of the topologies, the current stress of every single IGBT module is different. Therefore, according to different current stresses, FZ1200R12HE4 IGBT modules [34] considered for the primary IGBT modules of the proposed converter; FZ1600R12HP4 and FZ400R12KE4 IGBT modules [34] considered for the primary IGBT modules of unidirectional cascaded ISOS and IPOS converters, respectively. Meanwhile, due to the similar configuration at secondary side of all three topologies, D1131SH diodes [35] is selected for all of them. Table III shows the parameters of selected IGBTs/Diode.

The losses of the medium frequency transformer are calculated based on the parameters tabulated in Table IV, which are reported in [36]. The power losses and efficiency of the different topologies are illustrated in Fig.9 and Fig.10, respectively. As expected, the proposed converter shows a better performance in many aspects compared with unidirectional ISOS and IPOS, especially, in terms of losses and components utilization. From Fig.9 (a), it can get that the total losses of the proposed converter are much smaller than the ISOS converter but slightly bigger than the IPOS converter. However, the number of employed IGBT module of the IPOS converter is much bigger than the proposed converter and ISOS converter as shown in Fig.9(b), which would complicate the control system, hence decrease the reliability.

Table II System parameters of the DC/DC converters

\begin{tabular}{|l|c|c|c|}
\hline \multicolumn{1}{|c|}{ Items } & $\begin{array}{l}\text { Proposed } \\
\text { Converter }\end{array}$ & $\begin{array}{l}\text { Conventional } \\
\text { Unidirectional } \\
\text { ISOS } \\
\text { Converters }\end{array}$ & $\begin{array}{l}\text { Conventional } \\
\text { Unidirectional } \\
\text { IPOS } \\
\text { Converters }\end{array}$ \\
\hline Rated power & \multicolumn{3}{|c|}{$10 \mathrm{MW}$} \\
\hline Input DC voltage & \multicolumn{3}{|c|}{$15 \mathrm{kV}$} \\
\hline Output DC voltage & \multicolumn{3}{|c|}{$140 \mathrm{kV}$} \\
\hline $\begin{array}{l}\text { Total number of } \\
\text { IGBT modules at the } \\
\text { primary side }\end{array}$ & $\begin{array}{c}112 \\
(28 * 4)\end{array}$ & $\begin{array}{c}56 \\
(14 * 4)\end{array}$ & $(14 * 4 * 14)$ \\
\hline $\begin{array}{l}\text { Primary rated IGBT } \\
\text { module voltage }\end{array}$ & \multicolumn{3}{|c|}{$1.07 \mathrm{kV}$} \\
\hline $\begin{array}{l}\text { Total number of } \\
\text { Diode modules at } \\
\text { the secondary side }\end{array}$ & $(14 * 8)$ \\
\hline $\begin{array}{l}\text { Primary rated } \\
\text { submodule voltage }\end{array}$ & \multicolumn{3}{|c|}{$1.07 \mathrm{kV}$} \\
\hline $\begin{array}{l}\text { Secondary rated } \\
\text { diode voltage }\end{array}$ & \multicolumn{3}{|c|}{$5 \mathrm{kV}$} \\
\hline Modulation method & Sinusoidal pulse width modulation $(\mathrm{SPWM})$ \\
\hline $\begin{array}{l}\text { carrier-reference } \\
\text { frequency ratio }\left(R_{c}\right)\end{array}$ & \multicolumn{3}{|c|}{$f_{M} / f_{c}=5$} \\
\hline AC frequency $\left(f_{C}\right)$ & \multicolumn{3}{|c|}{$400 \mathrm{HHz}$} \\
\hline $\begin{array}{l}\text { Switching frequency } \\
\left(f_{M}\right)\end{array}$ & \multicolumn{3}{|c|}{} \\
\hline
\end{tabular}

Table III Parameters of IGBT/Diode

\begin{tabular}{|l|l|l|}
\hline IGBT/Diode Code & Rated Voltage & Rated Current \\
\hline FZ1200R12HE4 & $1.2 \mathrm{kV}$ & $1.2 \mathrm{kA}$ \\
\hline FZ1600R12HP4 & $1.2 \mathrm{kV}$ & $1.6 \mathrm{kA}$ \\
\hline FZ400R12KE4 & $1.2 \mathrm{kV}$ & $0.4 \mathrm{kA}$ \\
\hline D1131SH & $6.5 \mathrm{kV}$ & $1.1 \mathrm{kA}$ \\
\hline
\end{tabular}

Table IV Parameters of the medium frequency transformers

\begin{tabular}{|c|c|c|c|}
\hline Items & $\begin{array}{l}\text { Transformer } \\
\text { for proposed } \\
\text { Converter }\end{array}$ & $\begin{array}{l}\text { Transformer } \\
\text { for } \\
\text { unidirectional } \\
\text { ISOS } \\
\text { Converters }\end{array}$ & $\begin{array}{l}\text { Transformer } \\
\text { for } \\
\text { unidirectional } \\
\text { IPOS } \\
\text { Converters }\end{array}$ \\
\hline $\begin{array}{l}\text { Required } \\
\text { transformer } \\
\text { numbers }\end{array}$ & 1 & 14 & 14 \\
\hline Rated power & $10 \mathrm{MW}$ & $0.8 \mathrm{MW}$ & $0.8 \mathrm{MW}$ \\
\hline Rated frequency & $400 \mathrm{~Hz}$ & $400 \mathrm{~Hz}$ & $400 \mathrm{~Hz}$ \\
\hline $\begin{array}{ll}\begin{array}{l}\text { Rated } \\
\text { voltage }\end{array} & \text { primary } \\
\end{array}$ & $10 \mathrm{kV}$ & $1.1 \mathrm{kV}$ & $10 \mathrm{kV}$ \\
\hline $\begin{array}{ll}\text { Rated } & \text { secondary } \\
\text { voltage } & \\
\end{array}$ & $140 \mathrm{kV}$ & $10 \mathrm{kV}$ & $10 \mathrm{kV}$ \\
\hline $\begin{array}{l}\text { Primary equivalent } \\
\text { resistance }\end{array}$ & $5.2 \mathrm{~m} \Omega$ & $0.66 \mathrm{~m} \Omega$ & $54.29 \mathrm{~m} \Omega$ \\
\hline $\begin{array}{l}\text { Secondary } \\
\text { equivalent } \\
\text { resistance }\end{array}$ & $580 \mathrm{~m} \Omega$ & $54.29 \mathrm{~m} \Omega$ & $54.29 \mathrm{~m} \Omega$ \\
\hline Core material & \multicolumn{3}{|c|}{ Magnetic alloy 2605SA1 [35] } \\
\hline $\begin{array}{l}\text { Core coefficients } \\
\text { for Steinmetz } \\
\text { Equation } \\
\end{array}$ & \multicolumn{3}{|c|}{$k=1.4, \alpha=1.47, \beta=1.52$} \\
\hline $\begin{array}{ll}\begin{array}{l}\text { Saturated } \\
\text { density }\end{array} & \text { flux } \\
\end{array}$ & \multicolumn{3}{|c|}{$1.56 \mathrm{~T}$} \\
\hline Core volume & $0.21 \mathrm{~m}^{3}$ & $0.033 \mathrm{~m}^{3}$ & $0.033 \mathrm{~m}^{3}$ \\
\hline
\end{tabular}



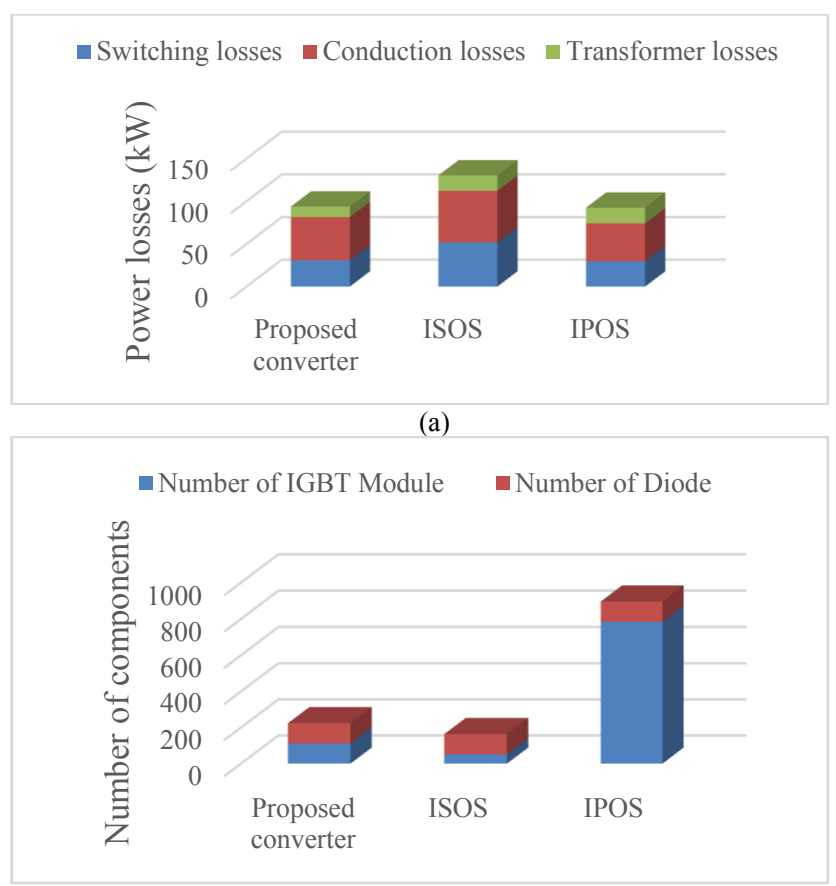

(b)

Fig.9 (a) Distribution of the power losses and (b) the number of employed IGBT/Diode of the proposed converter, ISOS and IPOS converters for operation at rated power $10 \mathrm{MW}$.

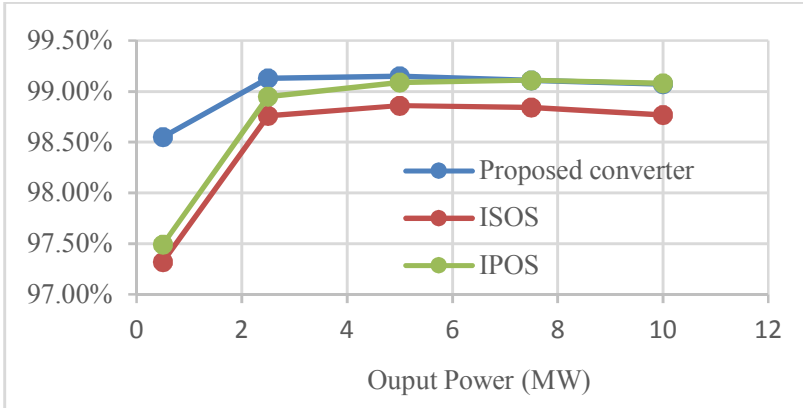

Fig.10 Efficiency of the proposed converter (blue line), ISOS (red line) and IPOS (green line) converters based on different rated power.

\section{The Overall Control of the Proposed CONVERTER}

\section{A. Implementation of the closed loop control}

The MMC converter in the proposed configuration operates as a voltage source to produce constant amplitude and frequency $\mathrm{AC}$ voltage to control the output voltage and current produced by the cascaded diode bridge rectifiers to facilitate the employment of the HVDC transmission lines. Therefore, this section employed a stationary frame regulator, the Proportional Resonant (PR) regulator, which achieves the same transient and steady-state performance as a synchronous frame PI regulator [37]. The proposed regulator is applicable to the single-phase system. Considering single-phase MMC as an example, the implementation of the employed closed loop control is illustrated in Fig.11. It is realized by multiplying the transfer functions of the conventional Proportional Integral $\left(\mathrm{PI}_{1}\right.$ and $\left.\mathrm{PI}_{2}\right)$ regulators with the sin and cos reference signals. This is applicable for both, steady-state and transient operations as the PI regulator operates under the $d-q$ synchronous rotating reference frame [37]. It is worth noting that there is no Park transformation or any other transformations between the stationary and rotating reference frame involved. Equation (82) describes the implementation



of the employed control method, which is schematically depicted in Fig. 11.

$V_{a c}^{r e f}(\mathrm{t})=\left\{\left[e_{a c}(t) \cdot \sin (\omega t)\right] * h_{d c}(t)\right\} \cdot \sin (\omega t)+$

$\left\{\left[e_{a c}(t) \cdot \cos (\omega t)\right] * h_{d c}(t)\right\} \cdot \cos (\omega t)$

where $\mathrm{e}_{a c}(t)$ is the input AC error signal, $h_{d c}(t)$ represents the unit impulse response under time domain of PI regulator, and ' $*$ ' donates the convolution product. The output of this control loop $V_{a c}^{r e f}(t)$ is used as a modulating signal to drive the power switches.

Actually, after multiplying with reference signal, sine and cos, the error signal $e_{a c}(t)$ is converted into a DC and $\mathrm{AC}$ components with two times the fundamental frequency. This is then fed into the PI regulators, which perform the integration to get the steady-state error and also work as a low-pass filter to extract out the DC signal to achieve a zero steady-state error in the stationary reference frame. Applying Laplace transform to (82), yields:

$$
V_{a c}^{r e f}(s)=\frac{1}{2}\left[H_{d c}(s+j \omega)+H_{d c}(s-j \omega)\right] e_{a c}(s)
$$

For the conventional PI controller, the Laplace transform of a unity impulse response can be described by the following equation:

$$
H_{d c}(s)=k_{p}+\frac{k_{i}}{s}
$$

By substituting (84) into (83), the transfer function of the control system with derived generalized integrator can be given as:

$$
V_{a c}^{r e f}(s)=\left(k_{p}+\frac{k_{i} \cdot s}{s^{2}+\omega^{2}}\right) e_{a c}(s)
$$

where $k_{p}$ is the proportional constant which is used to improve the transient response of the control system, $k_{i}$ is the integral constant and $\omega$ is the resonant frequency of the derived integrator. Therefore, the infinity gain can be achieved when $s=j \omega$.

\section{B. Theoretical verification of the derived generalized integrator}

To simplify the theoretical verification, the proposed converter is approximated as an MMC with a simple $R L$ load ( $L_{s}$ and $R_{S}$ ), where the MMC is represented by a unit gain. Fig. 12 shows the resultant simplified control system (i.e.in $S$ domain). Since the proposed converter is controlled via the primary MMC only, therefore, the AC voltage is fed back from the primary side of the transformer and compared with its reference value, $V_{a c}^{r e f}$. Then, the resultant $\mathrm{AC}$ voltage error, $e_{a c 1}$ is divided by the equivalent load ( $L_{s}$ and $R_{s}$ ) in the circuit to get the AC current error $e_{a c 2}$, which is used as an input to the generalized integrator, $H(s)$. The output of the $H(s)$ functions as the reference signal of the primary $\mathrm{AC}$ voltage. According to Fig.12, the transfer function of the MMC voltage controller from $V_{a c}^{r e f}(s)$ to $V_{a c}(s)$ can be expressed as:

$$
\frac{V_{a c}}{V_{a c}^{r e f}}=\frac{H(s)}{H(s)+\left(s L_{s}+R_{S}\right)}
$$

where $V_{a c}(s)$ and $V_{a c}^{r e f}(s)$ are the output and the reference 




Fig.12. Simplified close loop control block diagram for the primary MMC of the proposed DC/DC converter.

voltages of the MMC, respectively and $H(\mathrm{~s})$ is the transfer function of the generalized integrator, which is given by

$$
H(\mathrm{~s})=k_{p}+\frac{2 k_{i} \cdot s}{s^{2}+\omega^{2}}
$$

where $\omega$ is set to the fundamental frequency.

Substituting $s=j \omega$ into (87) yields;

$$
H(\mathrm{~s})=k_{p}+\frac{2 k_{i} \cdot s}{(j \omega)^{2}+\omega^{2}} \rightarrow+\infty
$$

Substituting (88) into (86) yields the characteristics of the proposed controller at the fundamental frequency $\omega$.

$$
\frac{V_{a c}(j \omega)}{V_{a c}^{r e f}(j \omega)}=1
$$

In order to get a pure sinusoidal $V_{a c}$ without harmonic distortion, the input voltage reference $V_{a c}^{r e f}$ is set to the fundamental frequency component only. Furthermore, (89) reveals that the actual output voltage $V_{a c}(j \omega)$ exactly matches its reference value, $V_{a c}^{r e f}(j \omega)$. This confirms that the proposed voltage controller can successfully achieve zero steady-state error at the fundamental frequency.

\section{Simulation AND EXPERIMENTAL RESUlTS}

\section{A. Simulation results}

A simulation model of the proposed converter rated at 10 $\mathrm{MW} / 140 \mathrm{kV}$ is developed with the tabulated parameters in Table $\mathrm{V}$ using MATLAB/SIMULINK to validate the feasibility and the effectiveness of the proposed system and its control system performance. In this paper, the MMC at the primary side is constructed using fourteen half-bridge SMs per arm and there are fourteen series-connected diode-bridge rectifier modules at the secondary side of the transformer. Furthermore, the medium frequency transformer with turn ratios of $W_{p}: W_{s 1}: W_{s 2}: \cdots: W_{s 14}=1: 1: 1: \cdots: 1$ is chosen, where $W_{p}$ and $W_{s 1}$ to $W_{s 14}$ are the primary winding and the corresponding secondary winding-one to winding-fourteen, respectively. However, different turns ratio can be also considered for different stepping gain if required.

The steady-state voltage and current of the proposed converter, operating at $400 \mathrm{~Hz}$ is depicted in Fig. 13. Since the proposed converter uses MMC at the primary side and diode bridge rectifier modules at the secondary side, the resultant primary terminal voltage, $V_{a b}$ and secondary voltages as shaped as multilevel and square waveforms (taking $V_{s 1}$ and $V_{s 2}$ as exmaple), respectively.

To demonstrate the effect of unbalance phenomena between the MMC arms, Figs. 14 and 15 show the voltage, current and instantaneous power of the upper and lower arms for both, balanced $(\mathrm{k}=0)$ and unbalanced $(\mathrm{k}=0.08)$ cases, respectively. Fig. 15 shows that the upper arm voltage is charged to a higher-level due to the introduced positive unbalanced factor. Meanwhile, the arm current and the instantaneous power flow become unbalance which will seriously influence the converter operation.

The performance of the voltage balance control is demonstrated in Fig.16, where it can be clearly observed that, the capacitor voltages are perfectly controlled, and closed to one-fourteenth of the input DC voltage (i.e. $15 \mathrm{kV} / 14$ ).


Fig.13. (a) Transformer primary terminal voltage waveform $V_{a b}$, (b) transformer secondary winding-one voltage waveform $V_{s 1}$, (c) transformer secondary winding-two voltage waveform $V_{S 2}$, (d) transformer primary current waveform $I_{P}$,(e) transformer secondary winding-one current waveform $I_{S 1}$, (f) transformer secondary winding-two current waveform $I_{S 2}$.

The output DC voltage of the proposed converter is shown in Fig. 17 and with the given transformer turns ratio in this paper $\left(W_{p}: W_{s 1}: W_{s 2}: \cdots: W_{s 14}=1: 1: 1: \cdots: 1\right)$, the average output DC voltage is maintained around $140 \mathrm{kV}$.
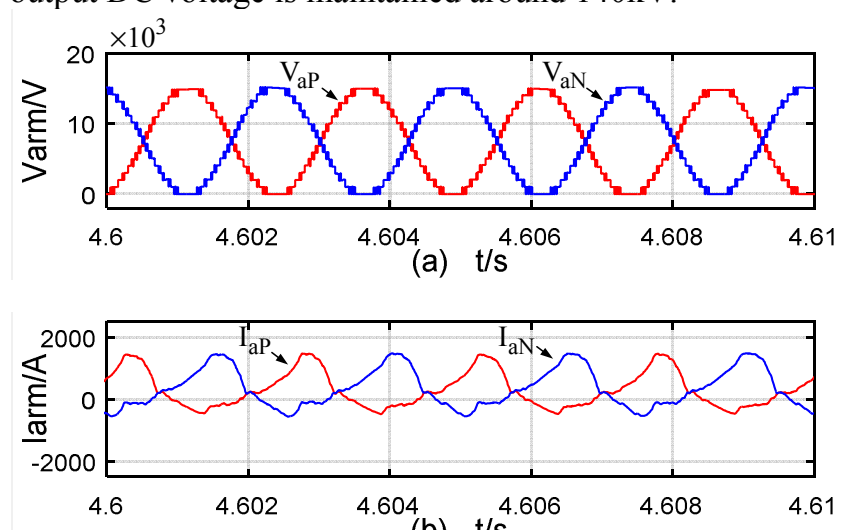

(b) $t / s$ 


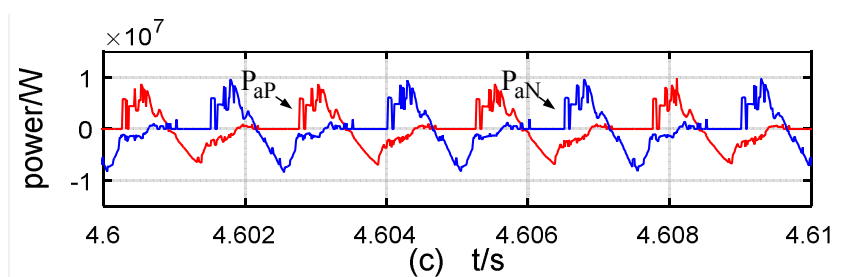

Fig.14. The primary side MMC key-waveforms with balanced state $\mathrm{k}=0$ : (a) upper arm voltage $V_{a P}$ and lower arm voltage $V_{a N}$, (b) upper arm current $I_{a P}$ and lower arm current $I_{a N}$, (c) upper arm power $P_{a P}$ and lower arm power $P_{a N}$.

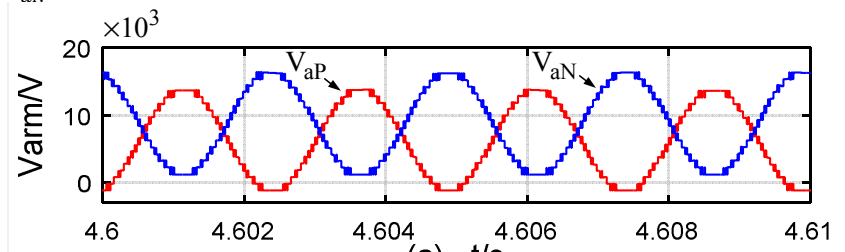

(a) $t / s$

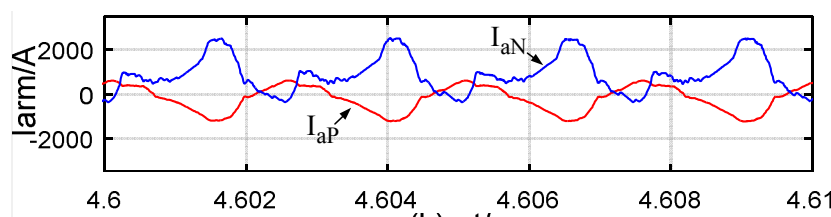

(b) $\mathrm{t} / \mathrm{s}$

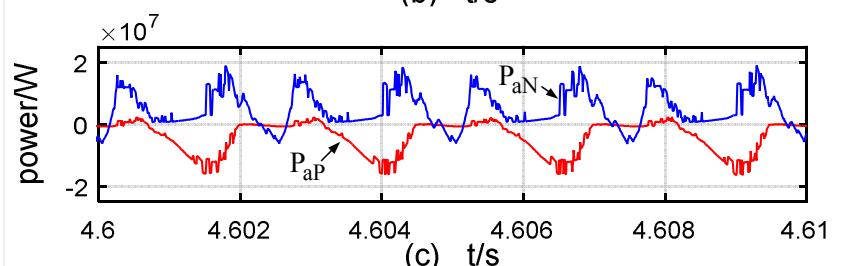

Fig.15. The primary side MMC key-waveforms with unbalanced state $\mathrm{k}=0.08$ : (a) upper arm voltage $V_{a P}$ and lower arm voltage $V_{a N}$, (b) upper arm current $I_{a P}$ and lower arm current $I_{a N}$, (c) upper arm power $P_{a P}$ and lower arm power $P_{a N}$.
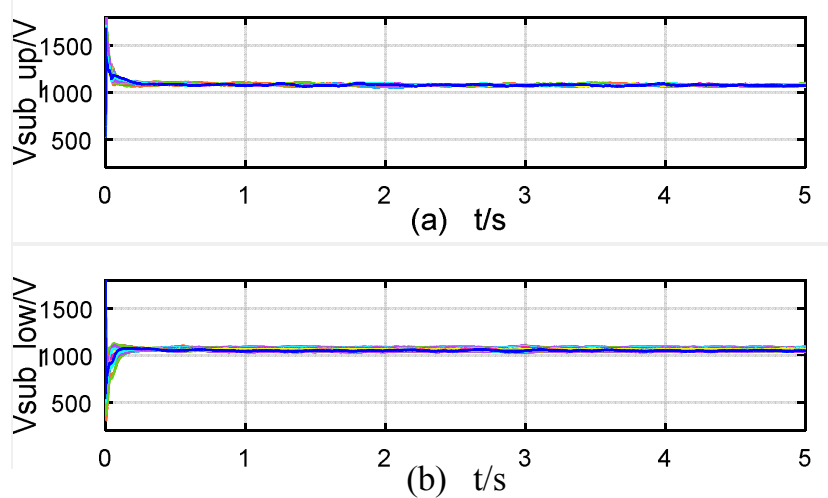

Fig.16. Upper and lower submodule voltages.
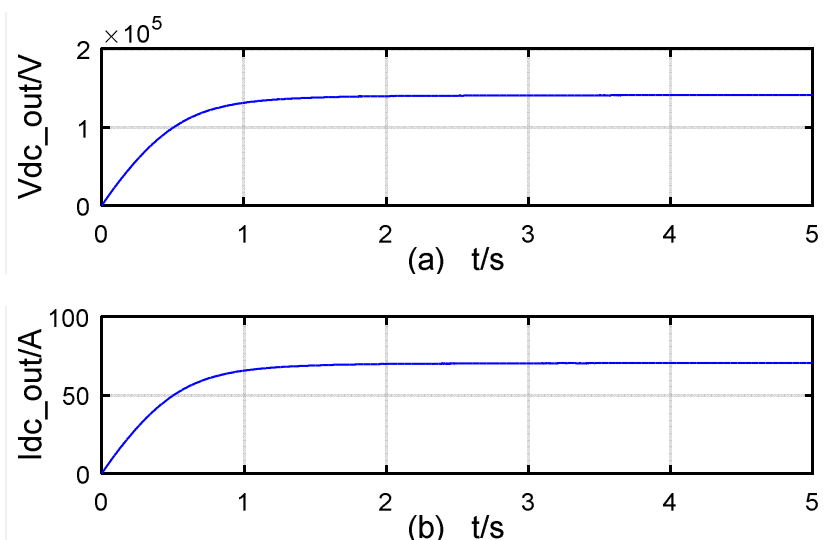

Fig.17. The output waveforms of the proposed converter under steady-state operation: (a) output DC voltage, (b) output DC current.
The performance of the employed control strategy is further investigated and confirmed with a step change in the output load ((i.e. $2 \mathrm{k} \Omega$ to 2.4k $\Omega$ ). Fig. 18 shows the dynamic response of the controller when the load changed at $t=2.5 \mathrm{~s}$, causing the output current $I_{d c 2}$ to decrease from $70 \mathrm{~A}$ to $60 \mathrm{~A}$, however the output voltage is perfectly maintained constant, which confirms the effectiveness of the control system.

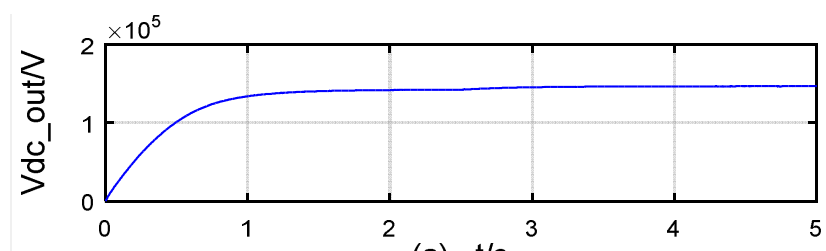

(a) $t / s$

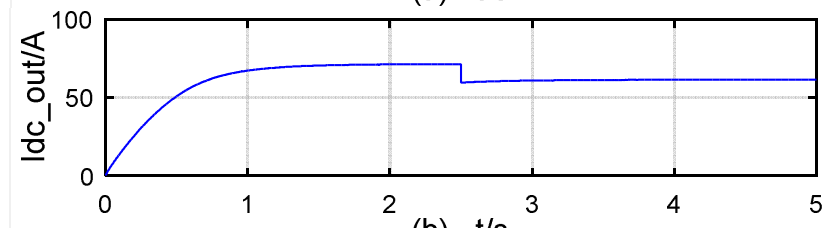

Fig.18. The output waveforms of the proposed converter under a step change at $2.5 \mathrm{~s}$ : (a) output DC voltage, (b) output DC current.

Table V Parameters of the simulated system
\begin{tabular}{|l|c|}
\hline Parameter & Value \\
\hline Rated power & $10 \mathrm{MW}$ \\
\hline Input DC voltage & $15 \mathrm{kV}$ \\
\hline Output DC voltage & $140 \mathrm{kV}$ \\
\hline Output load resistor & $2000 \Omega$ \\
\hline Number of MMC's SMs per arm & 14 \\
\hline Number of diode-bridge rectifier modules & 14 \\
\hline Transformer ratio & $1: 14$ \\
\hline SM capacitor of MMC & $2.2 \mathrm{mF}$ \\
\hline Arm Inductor & $1 \mathrm{mH}$ \\
\hline Output capacitor & $3 \mathrm{mF}$ \\
\hline Output inductor & $1 \mathrm{mH}$ \\
\hline Switching frequency & $2 \mathrm{kHz}$ \\
\hline AC fundamental frequency & $400 \mathrm{~Hz}$ \\
\hline
\end{tabular}

\section{B. Experimental validation}

Low voltage scaled down laboratory prototype is developed to validate and confirm the simulation and the theoretical studies of the proposed converter and the performance of the control strategies. Table VI summarizes the main parameters of the test-rig. The scaled down laboratory prototype and its schematic diagram are illustrated in Fig.19. It is worth noting that the proposed converter is intended for high-voltage highpower applications; therefore, the simulation is presented for close-to-reality systems. However, implementing such a system in the laboratory is not feasible from the safety and the resources point of view, therefore scaled down prototype is developed as a proof of concept and to validate the proposed control methods and their effectiveness.

The scaled down prototype is developed with the following considerations:

- The one-leg MMC is used for the primary side of the test-rig, instead of using single-phase MMC, (e.g., two-legs) as shown in the simulation. This will help to reduce the number of submodules (only $3 \mathrm{SMs}$ ) and the control complexity of the test-rig but satisfy the validation of the proposed topology and its control strategy.

- Likewise, as the proposed converter is modular at both sides, only 2 diode-bridge rectifier models are considered at the secondary side, which is enough to accommodate the requirement of the designed 
prototype. Once again, due to the modularity feature, this can be easily extended as presented in the simulation, if resources are available.

- As it can be seen from Fig.19, the test-rig is developed using the six drive circuit boards, seven voltage sensors, three current sensors, A/D SampleInterface board, and TMS320F28335DSP control board. However, in real applications, where a large number of submodules is required, different arrangement maybe needed, which include a higher number of control boards, more sophisticated controllers such as DSP, FPGA, and CPLD to meet these requirements. In general, most of large-scale control systems are built by the company, such as $\mathrm{ABB}$ and SIEMENS.

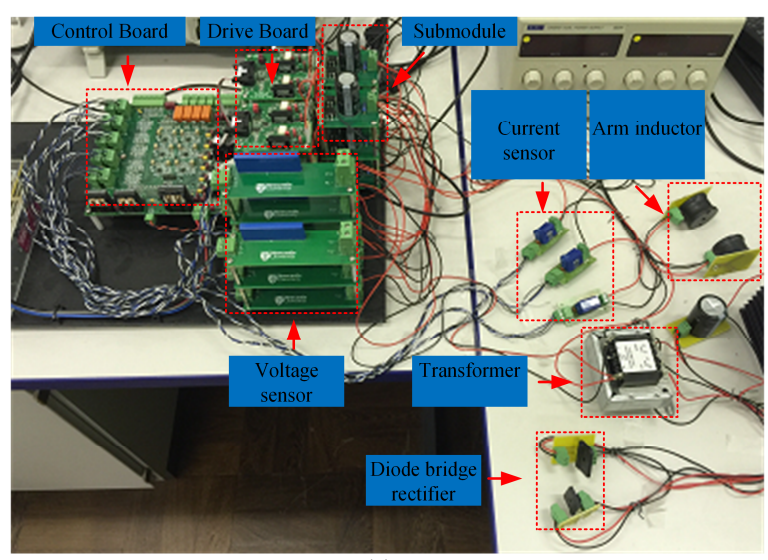

(a)



(b)

Fig .19 (a)Scaled down laboratory prototype and its (b) schematic diagram.

Fig.20 shows the steady-state performance of the proposed converter. The $400 \mathrm{~Hz}$ AC voltages at primary and secondary are shown in Fig. 20(a), and the corresponding currents at primary and the secondary side windings are depicted in Fig.20(b). It is worth noting that the peak of secondary currents is just half of the primary one (i.e. $\frac{I_{p_{-} p e a k}}{2}=$ $\left.I_{s 1 \_p e a k}=I_{s 2 \_p e a k}=4.1 \mathrm{~A}\right)$, which effectively reduce the current rating of the associated diode bridge rectifier submodules. This can be further reduced if more windings on the secondary side are considered. The Root Mean Square $(R M S)$ value of the primary terminal voltage is stepped up by a transformer with turn ratios of $W_{p}: W_{s 1}: W_{s 2}=1: 1: 1$ from $V_{a b_{-} R M S}=\frac{30 \mathrm{~V}}{\sqrt{2}}=21 \mathrm{~V}$ to $V_{S_{-} R M S}=V_{S 1_{-} R M S}+V_{S 2_{-} R M S}=$ $19 \mathrm{~V}+19 \mathrm{~V}=38 \mathrm{~V}$, (noting that: the $R M S$ value of the square waveform is equal to the peak value). Furthermore, the current and voltage stresses of the diode bridge rectifier modules will be further reduced if higher number of modules are considered. Fig. 20(c) shows the experimental output DC voltage and current waveforms of the proposed converter, which are perfectly smooth and regulated at the required value, confirming the effectiveness of the proposed control system.

Fig.21 shows the performance of the submodule voltage balance control, where it can be seen that the capacitor voltages are well balanced and closed to one third of the DC input voltage (i.e. 23V). Meanwhile, the voltage ripple of each submodule is relatively small.

The dynamic performance of the system is experimentally validated as well, a step change to the load is applied causing the output power to decrease from $84 \mathrm{~W}$ to $44 \mathrm{~W}$. This can be clearly seen from Fig.22, where the primary peak current changes from $I_{p_{-} p e a k}= \pm 8.2 \mathrm{~A}$ (peak value of primary current) to $I_{p_{-} \text {peak }}= \pm 4.5 \mathrm{~A}$ (peak value of primary current after a step change) as a response to the variations of the output side load. At the same time, primary peak terminal voltage, $V_{\text {ab_peak }}$ remains controlled at $\pm 30 \mathrm{~V}$, which effectively demonstrates the excellent performance of the primary voltage control strategy. Consequently, the output DC current quickly decreased from $2.1 \mathrm{~A}$ to $1.1 \mathrm{~A}$ and DC voltage remains at $40 \mathrm{~V}$, as illustrated in Fig. 22 (b). This further verify the effectiveness of the proposed system and its control strategy.



(a) $(2 \mathrm{~ms} /$ div $)$



(b) $(2 m s / d i v)$

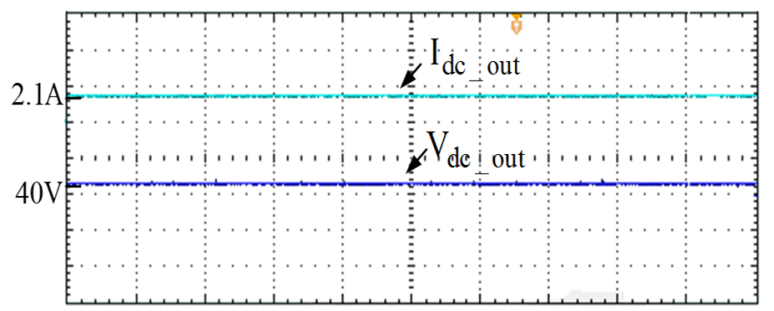

(c) $(10 \mathrm{~ms} /$ div $)$

Fig.20 Under steady state, (a) transformer primary terminal voltage $V_{a b}(40 \mathrm{~V} / \mathrm{div})$, secondary winding-one voltage $V_{s 1}$, and winding-two voltage $V_{s 2}$ (20V/div), (b) transformer primary current $I_{P}(10 \mathrm{~A} /$ div $)$, secondary winding-one current $I_{s 1}$, and winding-two current $I_{s 2}(5 \mathrm{~A} / \mathrm{div})$, (c) proposed converter output DC current $I_{d c \_o u t}(5 \mathrm{~A} / d i v)$ and voltage $V_{d c_{-} \text {out }}(40 \mathrm{~V} / d i v)$. 


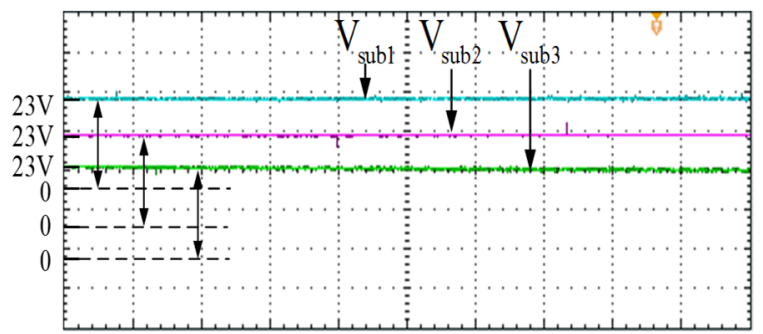

(a) $(10 \mathrm{~ms} / \mathrm{div})$

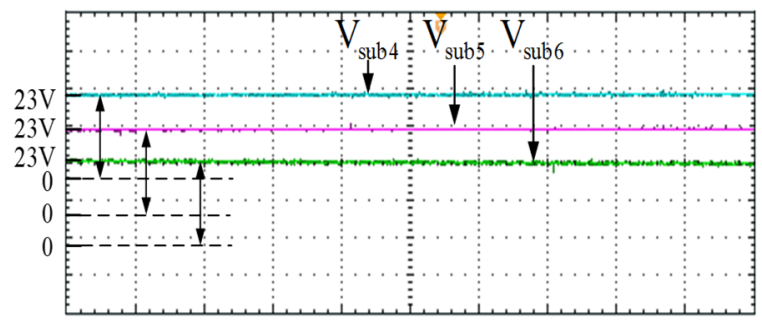

(b) $(10 \mathrm{~ms} /$ div $)$

Fig.21 Under steady state, (a) upper arm voltages $V_{\text {sub } 1}, V_{\text {sub } 2}$ and $V_{\text {sub } 3}$ (10V/div), (b) lower arm submodule voltages $V_{s u b 4}, V_{s u b 5}$ and $V_{s u b 6}$ (10V/div).

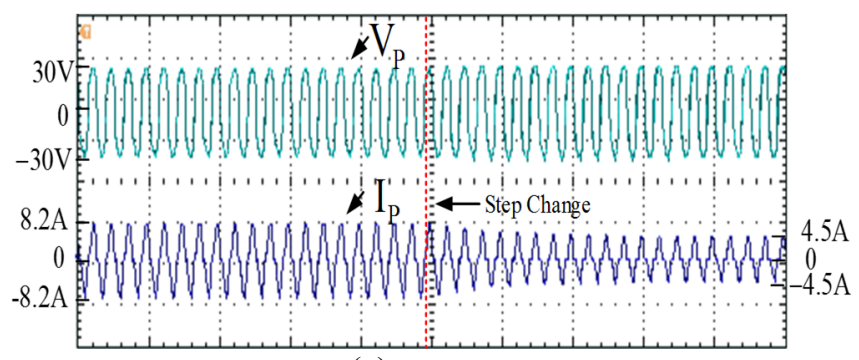

(a) $(10 \mathrm{~ms} /$ div $)$



(b) $(10 \mathrm{~ms} / \mathrm{div})$

Fig.22 Dynamic response, (a) transformer primary terminal voltage $V_{a b}$ (30V/div) and current $I_{P}(10 \mathrm{~A} / d i v)$, (b) proposed converter output DC voltage $V_{d c_{-} \text {out }}(40 \mathrm{~V} / \mathrm{div})$ and current $I_{\text {dc_out }}(1 \mathrm{~A} / \mathrm{div})$.

Table VI Parameters of the experimental setup
\begin{tabular}{|l|c|}
\hline Parameter & Value \\
\hline Rated power & $80 \mathrm{~W}$ \\
\hline Input DC voltage & $70 \mathrm{~V}$ \\
\hline Output load resistor & $20 \Omega$ \\
\hline Primary side AC peak voltage of transformer & $\pm 35 \mathrm{~V}$ \\
\hline Submodule numbers of MMC per arm & 3 \\
\hline Submodule numbers of combined converter & 2 \\
\hline Transformer ratio & $1: 1: 1$ \\
\hline Submodule capacitor of MMC & $2.2 \mathrm{mF}$ \\
\hline Inductance of per arm & $1 \mathrm{mH}$ \\
\hline Output capacitor & $3 \mathrm{mF}$ \\
\hline Output inductor & $1 \mathrm{mH}$ \\
\hline Switching frequency & $2000 \mathrm{~Hz}$ \\
\hline AC frequency & $400 \mathrm{~Hz}$ \\
\hline Sampling rate (F28335) & $20 \mathrm{kHz}$ \\
\hline
\end{tabular}

\section{CONCLUSION}

A modular unidirectional DC/DC converter based offshore DC collecting point is presented in this paper. The proposed converter utilized the state-of-the-art MMC at the primary side of a medium frequency transformer and cascaded diodebridge rectifier modules at the secondary side. The converter design features modularity, expandability, galvanic isolation, less losses, lower device voltage and current ratings, and higher efficiency. Detailed theoretical design analysis is presented and parameters that effect the operation of the converter are defined and thoroughly discussed. This includes the energy management between the MMC arms and submodules, power balancing, the power transfer capability of the converter, converter and transformer losses calculation, and comparative study with a competitive circuit topology. As expected, the proposed converter shows superior performance in terms of efficiency, losses and devices utilization, when compared with the most competitive unidirectional cascaded ISOS and IPOS converters, which makes it more attractive for this particular application. An advanced stationary frame regulator, the Proportional Resonant (PR) regulator, which achieves the same transient and steady-state performance as a synchronous frame PI regulator along with other sub-control loops including submodule voltage balance control and circulation current suppression is employed for the proposed converter. The employed control strategy abolishes all complexity associated with the multiple transformations required for the conventional $d-q$ synchronous reference frame methods. The employed generalized controller directly acts on the AC single of the primary side, avoiding complex transformation and providing robustness against system variations. The performance of the proposed converter and its control strategy is validated through various simulation and experimentally verified results.

\section{APPENDIX}

1) The parameter of the submodule capacitor is determined by [38] [39]:

$$
\mathrm{C}=\Delta E_{\text {arm }} / N \Delta V V_{\text {avg }}
$$

Where $C$ is the capacitance of the submodule capacitor; $\Delta E_{\text {arm }}$ is the energy variations in the arms; $N$ is submodule number per arm; $\Delta V$ is voltage ripple of submodule capacitor; $V_{\text {avg }}$ is average voltage of submodule capacitor.

2) The parameter of the arm inductor is determined by [40] [41]:

\section{A. Taking reducing fault current as criterion:}

$$
L_{\text {arm }}=\frac{N V_{\text {capacitor }}}{2 \mathrm{~K}}
$$

Where $K=\frac{d I_{a P}}{d t}=\frac{d I_{N}}{d t} ; V_{\text {capacitor }}$ is the submodule capacitor voltage; $N$ is the submodule number per arm.

$$
\begin{aligned}
& \text { B. Taking suppressing circulating current as criterion: } \\
& \qquad L_{\text {arm }}=\frac{1}{8 \omega^{2} \cdot C \cdot V_{\text {capacitor }}}\left(\frac{P_{S}}{3 I_{2} \omega}+V_{d c_{-} \text {in }}\right)
\end{aligned}
$$

Where $I_{2 \omega}$ is the allowed value of second order arm current component; $\omega$ is the fundamental angular frequency; $C$ is the submodule capacitance; $V_{\text {capacitor }}$ is the submodule capacitor voltage; $P_{S}$ is the apparent power of the converter. $V_{d c_{-} \text {in }}$ is the input DC voltage. 


\section{REFERENCES}

[1] P.Bresesti, W.L.Kling, R.L.Hendriks, R.Vailati, "HVDC connection of offshore wind Farms to the transmission system," IEEE Trans. Energy Convers., vol.22, pp.37-43, Mar.2007.

[2] J.Robinson, D.Jovcic, G.Joos, "Analysis and design of an offshore wind farm using a MV DC grid," IEEE Trans. Power Del., vol.25, pp.2164-2173, Oct.2010.

[3] C. Sun, J. Zhang, X. Cai, and G. Shi, "Voltage balancing control of isolated modular multilevel dc-dc converter for use in dc grids with zero voltage switching," IET Power Electron., vol.9, pp.270280, Feb.2016.

[4] J. Everts, "Closed-Form solution for efficient ZVS modulation of DAB converters," IEEE Trans. Power Electron., vol.32, pp.75617576, Oct. 2017.

[5] W. Chen, X. Ruan, H. Yan, and C. Tse, "DC/DC conversion system consisting of multiple converter modules: stability, control, and experimental verifications," IEEE Trans. Power Electron., vol.24, pp.1463-1474, Jun. 2009.

[6] T. Li, and L. Parsa, "Design, control and analysis of a fault tolerant soft-switching DC-DC converter for high power high voltage applications," IEEE Trans. Power Electron., vol.33, pp.1094-1104, Mar. 2017.

[7] Y. Lian, G. Adam, D. Holliday and S. Finney, "Medium-voltage DC/DC converter for offshore wind collection grid," IET Renewable Power Generation, vol.5, pp.651-660, April 2016.

[8] Y. Lian, G. Adam, D. Holliday and S. Finney, "Modular InputParallel Output-Series DC/DC converter control with fault detection and redundancy," IET Generation Trans. \& Distribution, vol.5, pp.1361-1369, May 2016.

[9] C.Zhong, J.Biao, J.Feng, S.Lei, "A novel ZVS full-bridge converter with auxiliary circuit," in Proc. IEEE Appl. Power Electron. Conf. Expos., Feb. 2010, pp.1448-1453.

[10] S. Y, Lin and C. L. Chen, "Analysis and design for RCD clamped snubber used in output rectifier of phase shifted full-bridge ZVS converters," IEEE Trans. Ind. Electron., vol. 45, no. 2, pp. 358359, Apr. 1998.

[11] G. Hua, F. C. Lee, M. M. Jovanovic, "An improved full-bridge zero-voltage-switched PWM converter using a saturable inductor," IEEE Trans. Power Electron., vol.8, pp.530-534, Oct.1993.

[12] A. Chub, O. Husev, and D. Vinnikov, "Input-parallel outputseries connection of isolated quasi-Z-source DC-DC converters," in Proc. Electron. Power Quality Supply Rel. Conf., 2014, pp.277-284.

[13] H. Daneshpajooh, M. Pahlevaninezhad, P. Jain, A. Bakhshai, “An efficient soft switched DC-DC converter for electric vehicles," in Proc. IEEE Appl. Power Electron. Conf. Expos., Mar. 2013, pp.1798-1803.

[14] A. Mousavi, P. Das, G. Moschopoulos, "A novel ZCS-PWM fullbridge converter with a simple active auxiliary circuit" in Proc. IEEE Appl. Power Electro. Conf. Expos., Mar. 2012, pp.12731277.

[15] Z. Chen, S. Liu, and L. Shi, "Improved zero-voltage-switching pulse width modulation full bridge converter with self-regulating auxiliary current," IET Trans. Power Electron., vol.6, pp.287296, Feb.2013.

[16] R. Zeng, L. Xu, and L. Yao, "DC/DC converters based on hybrid MMC for HVDC grid interconnection," in Proc. IET Int. conf. AC/DC Power Trans., Feb. 2015, pp.1-6.

[17] C. Sun, J. Zhang, X. Cai and G. Shi, "analysis and arm voltage control of isolated modular multilevel DC-DC converter with asymmetric branch impedance," IEEE Trans. Power Electron., vol.32, pp.5978-5990, Aug.2017.

[18] Z. Xing, X. Ruan, H. You, X. Yang, D.Yao, and C. Yuan, ”Softswitching operation of isolated modular DC/DC converters for application in HVDC Grids," IEEE Trans. Power Electron., vol.31, pp.2753-2766, Apr. 2016.

[19] S. Kenzelmann, A. Rufer, M. Vasiladiotis, D. Dujic, F. Canales, Y. R. and De Novaes, "A versatile DC-DC converter for energy collection and distribution using the Modular Multilevel Converter," in Proc. Europe. Conf. Power Electron. Appl., Aug. 2011, pp.1-10.

[20] T. Luth, M. C. Merlin, T. C. Green, F. Hassan, and C. D. Barker, "High-Frequency operation of a DC/AC/DC system for HVDC applications," IEEE Trans. Power Electron., vol.29, pp.41074115, Aug.2014.

[21] J.Zhang, Z. Wang, and S. Shao, "A Three-Phase modular multilevel DC-DC converter for power electronic transformer applications," IEEE Trans. Emerging Selected Topics Power Electron. vol.5, pp.140-150, Mar.2017.

[22] M. Hajian, J. Robinson, D. Jovcic, and B. Wu, "30 kW, $200 \mathrm{~V} / 900 \mathrm{~V}$, Thyristor LCL dc/dc converter laboratory prototype design and testing," IEEE Trans. Power Electron., vol.29, pp. 1094-1102, Mar.2014.

[23] N. Denniston, A. Massoud, S. Ahmed, and P. Enjeti, "Multiplemodule high-gain high-voltage dc-dc transformers for offshore wind energy systems," IEEE Trans. Ind. Electron., vol. 58, pp. 1877-1886, May 2011.

[24] W. Chen, A. Q. Huang, C. Li, G. Wang, and W. Gu, "Analysis and comparison of medium voltage high power $\mathrm{dc} / \mathrm{dc}$ converters for off-shore wind energy systems," IEEE Trans. Power Electron., vol. 28, pp.2014-2023, Apr.2013.

[25] Q. Tu, Z. Xu, and L. Xu, "Reduced switching-frequency modulation and circulating current suppression for modular multilevel converters," IEEE Trans. Power Electron., vol.29, pp. 1094-1102, Mar.2014.

[26] R. Doncker, D. Divan, and M. Kheraluwala, "A three-phase softswitched high-power-density DC/DC converter for high-power applications," IEEE Trans. Ind. Appl., vol.27, pp.63-73, Jan. 1991.

[27] A. Averberg and A. Mertens, "Characteristics of the single active bridge converter with voltage doubler," in Proc. 13th International Power Electron. And Motion Control Conf., Step. 2008, pp.213-220.

[28] R. Zeng, L. Xu, L. Yao, and B. W. Williams, "Design and operation of a hybrid modular multilevel converter," IEEE Trans. Power Electron., vol.30, pp.1137-1146, Mar. 2015.

[29] K. Ilves, S. Norrga, L. Harnefors, and H. Nee, "On energy storage requirements in modular multilevel converters," IEEE Trans. Power Electron., vol.29, pp.77-88, Jan.2014.

[30] M. Hagiware, and H. Akagi, "Control and Experiment of Pulse Width-Modulated Modular Multilevel Converters," IEEE Trans. Power Electron., vol.24, pp.1737-1746, Jul.2009.

[31] S. Rohner, S. Bernet, M. Hiller, and R. Sommer, "Modulation, losses, and semiconductor requirements of modular multilevel converters," IEEE Trans. Indus. Electron., vol.57, pp.2633-2642, Aug. 2010

[32] M. Amin Bahmani, T. Thiringer, and M. Kharezy, "Design methodology and optimization of a medium-frequency transformer for high-power DC-DC applications," IEEE Trans. Indus. Appl., vol.52, pp.4225-4233, Oct. 2016.

[33] C. C. Davidson and D. R. Trainer, "Innovative concepts for hybrid multi-level converters for HVDC power transformer," in Proc. Int. Conf. ACDC Power Trans., Oct. 2010, pp.1-5.

[34] Datasheet: IGBT module FZ1200R12HE4. [Online]. Available: www.infineon.com

[35] Datasheet: Diode D1131SH. [Online]. Available: www.infineon.com

[36] S. Meier, T. Kjellqvist, S. Norrga, and H. Nee, "Design considerations for medium-frequency power transformers in offshore wind farms," in Proc. 13th Europe. Conf. Power Electron. Appl., Sept. 2009, pp.1-12.

[37] D. N. Zmood, D. G. Holmes, "Stationary frame current regulation of PWM inverters with zero steady-state error," IEEE Trans. Power Electron., vol.18, pp.814-822, Aug.2003.

[38] K. Ilves, S. Norrga, L. Harnefors, and H. Nee, “On energy storage requirements in modular multilevel converters," IEEE Trans. Power Electron., vol.29, pp.7788, Jan.2014.

[39] J. Carr, D. Das, J. Li, J. Pan, S. Ebner, and O. Apeldoorn, "Modular multilevel converter for direct MVDC connection of offshore wind farms," IEEE Energy Conversion Congress and Exposition (ECCE), pp.976982, Sept.2015.

[40] Q.Tu, Z. Xu, R. Dacol, H. Huang, and J. Zhang, "Parameter design principle of the arm inductor in modular multilevel converter based HVDC," International Conf. Power System Technology, pp.1-6, Oct. 2010.

[41] L.Cunico, G. Lambert, R. Dacol, S. Oliveira, and Y. Novaes, "Parameters design for modular multilevel converter," Brazilian Power Electronics Conference, pp.264-270, Oct. 2013. 